\title{
L'aménagement durable dans les espaces ruraux mosellans à travers les exemples de Delme et Marsal
}

Sustainable development in rural areas of the Moselle département : the case studies of Delme and Marsal

Nachhaltige Raumordnung der ländlichen Gebiete in dem Departement Moselle: die Beispiele Delme und Marsal

\section{Fabien Gille}

\section{OpenEdition \\ Journals}

Electronic version

URL: http://journals.openedition.org/rge/5794

ISSN: 2108-6478

Publisher

Association des géographes de l'Est

\section{Printed version}

Date of publication: 22 April 2016

ISSN: 0035-3213

Electronic reference

Fabien Gille, "L'aménagement durable dans les espaces ruraux mosellans à travers les exemples de Delme et Marsal », Revue Géographique de l'Est [Online], vol.56 / n¹-2 | 2016, Online since 31 August 2016, connection on 08 September 2020. URL : http://journals.openedition.org/rge/5794

This text was automatically generated on 8 September 2020.

Tous droits réservés 


\title{
L'aménagement durable dans les espaces ruraux mosellans à travers les exemples de Delme et Marsal
}

\author{
Sustainable development in rural areas of the Moselle département : the case \\ studies of Delme and Marsal \\ Nachhaltige Raumordnung der ländlichen Gebiete in dem Departement Moselle: \\ die Beispiele Delme und Marsal
}

Fabien Gille

\section{Introduction}

Depuis la conférence de Rio en 1992, le développement durable est progressivement transposé dans l'aménagement du territoire français. L'Agenda 21 local est le document d'application. En France, il est présent dans plusieurs documents d'urbanisme (Plan Local d'Urbanisme, Schéma de Cohérente Territoriale par exemple). Les documents d'urbanisme ne sont pas les seuls outils d'aménagement durable. La multitude des projets à l'image des Zones d'Aménagement Concerté, des lotissements, des sentiers de valorisation de la nature, devient une nouvelle clef d'entrée pour l'urbanisme durable. Les premiers territoires ayant mis en œuvre ces outils sont les villes. Aujourd'hui, les campagnes, qui occupent la majorité du territoire français et qui connaissent une renaissance (Kayser B, 1990), se les approprient. Malheureusement, les communes rurales ne disposaient pas jusqu'à maintenant de la taille suffisante pour bien les faire évoluer. L'absence de techniciens locaux, leur manque de compétence ou encore la surcharge de travail des services déconcentrés de l'Etat ou des collectivités territoriales partenaires (région, département, parc naturel régional...) pesaient sur la mise en pratique d'un aménagement rural durable de qualité. La Lorraine est également concernée par cette problématique. 
2 Les bouleversements territoriaux influencent la place de l'aménagement durable dans les espaces ruraux lorrains. Cet article s'interroge sur la mise en œuvre de la durabilité dans la planification rurale française. Pour répondre à cette question, deux études de cas (Delme et Marsal en Moselle), aux enjeux différents, sont exposées. Ces deux communes sont presque voisines et se situent dans un département frontalier où l'exemple du voisin allemand peut être une source d'inspiration pour les projets ne s'inscrivant pas dans un cadre juridique précis.

3 L'article s'appuie sur l'analyse croisée du terrain et des documents de travail disponibles en mairie, dans les administrations associées aux différents projets d'aménagement (comptes rendus de réunions, échanges de courriers) et de la législation qui s'applique (code de l'urbanisme avec le Règlement National d'Urbanisme, carte communale, Plan Local d'Urbanisme, charte de Parc Naturel Régional ou de Pays...).

4 Après avoir présenté la place potentielle de la durabilité sur nos terrains d'étude, nous aborderons la transposition progressive du développement durable dans la législation française puis son application par l'intermédiaire de la multiplicité des acteurs et des réalisations locales concrètes.

\section{Présentation des terrains d'étude}

5 Le choix des communes de Delme et Marsal découle en premier lieu d'un travail méthodologique.

\section{A. Détermination des terrains d'étude}

6 La méthodologie s'appuie sur trois éléments. Le premier consiste à définir ce qu'est un espace rural pour donner une délimitation géographique de l'espace analysé. L'espace rural correspond aux territoires dont la Surface Agricole Utile (SAU) et la forêt représente plus de $80 \%$ de l'occupation du sol. Cette définition est couplée à celle de l'usage des espaces ruraux donnée par Philippe Perrier-Cornet (2002). Ce dernier distingue trois types d'usage :

7 - la «campagne cadre de vie » est un espace rural où la fonction résidentielle est importante

8 - la « campagne ressource » correspond au milieu rural où les productions agricoles et/ ou industrielles dominent

9 - la «campagne nature » correspond aux espaces ruraux où les documents de protection de la nature (Natura 2000 par exemple) sont présents

10 Une fois l'espace géographique délimité, nous nous appuyons sur une analyse statistique des communes rurales lorraines pour déterminer celles en forte croissance démographique.

11 L'objectif est d'effectuer une étude comparative entre deux territoires ruraux géographiquement proche qui s'inscrivent dans les mêmes limites administratives (communauté de communes, Pays, département, région) afin d'analyser la manière dont ces politiques supérieures sont appliquées sur deux territoires différents. 
Le recoupement de ces éléments montre l'intérêt particulier que présente Delme et Marsal. Delme connaît une croissance démographique exponentielle alors qu'elle se situe dans un espace rural. Elle fait à la fois partie, selon Philippe Perrier-Cornet, de la « campagne cadre de vie » et de la " campagne ressource ». Marsal est peu éloignée de Delme. Egalement situé en milieu rural, Marsal fait partie de la "campagne nature » et de la «campagne ressource». Elle s'inscrit également dans les mêmes limites administratives que Delme.

\section{B. Delme et Marsal, des communes rurales proches géographiquement mais différentes}

La Lorraine s'articule autour du Sillon Lorrain. Cet espace, qui s'étend d'Epinal aux portes du Luxembourg, regroupe la moitié de la population lorraine ${ }^{1}$. Le Sillon concentre également la plupart des emplois. La Moselle est le département le plus important démographiquement et économiquement de la région Lorraine.

Figure 1 : Dynamique socio-économique de la Lorraine

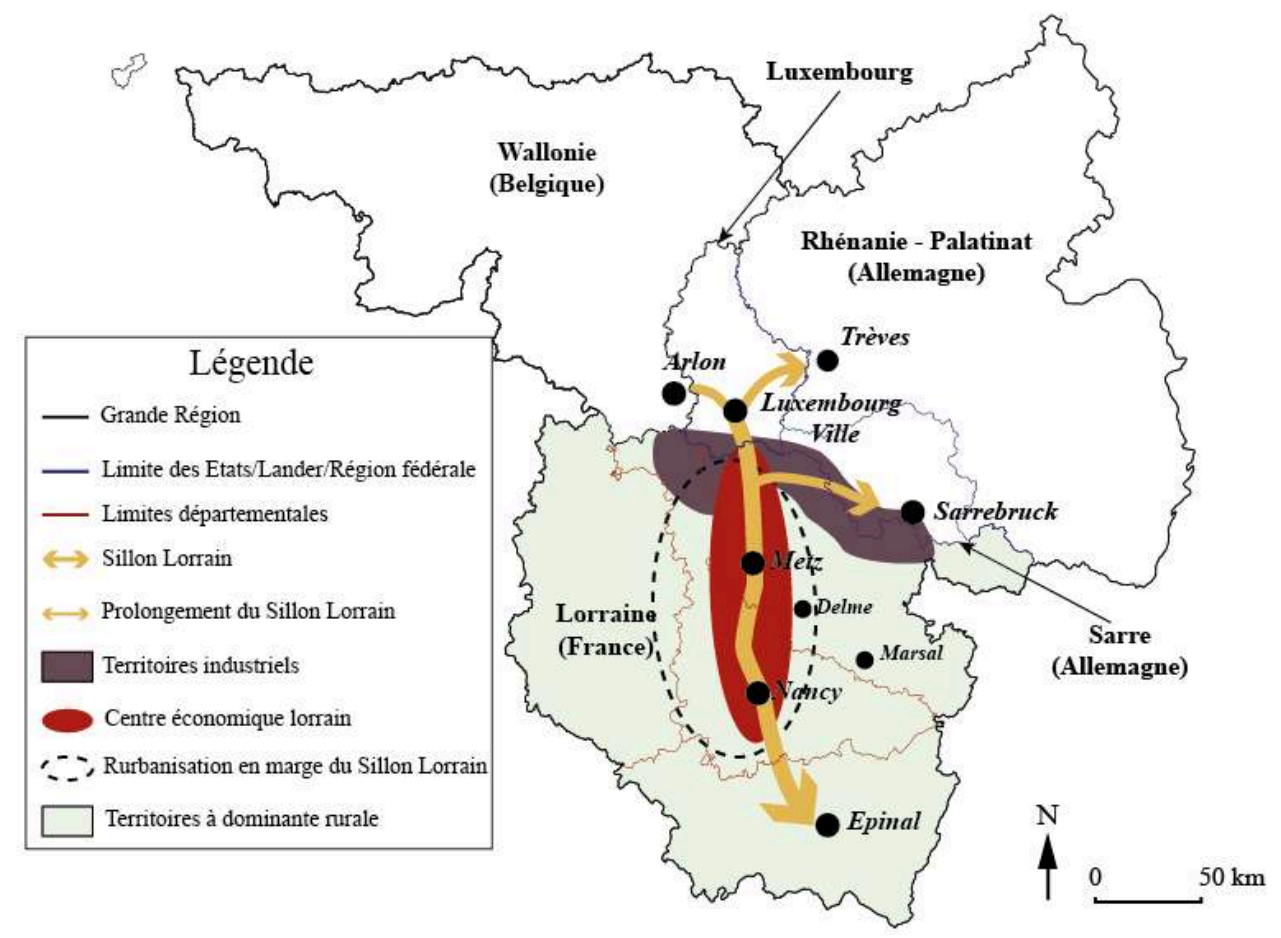

Conception : Gille, 2015

Delme et Marsal sont en marge du Sillon Lorrain (figure 2). 
Figure 2 : Delme et Marsal en marge et en périphérie du Sillon Lorrain

Marsal et Delme en marge et en périphérie

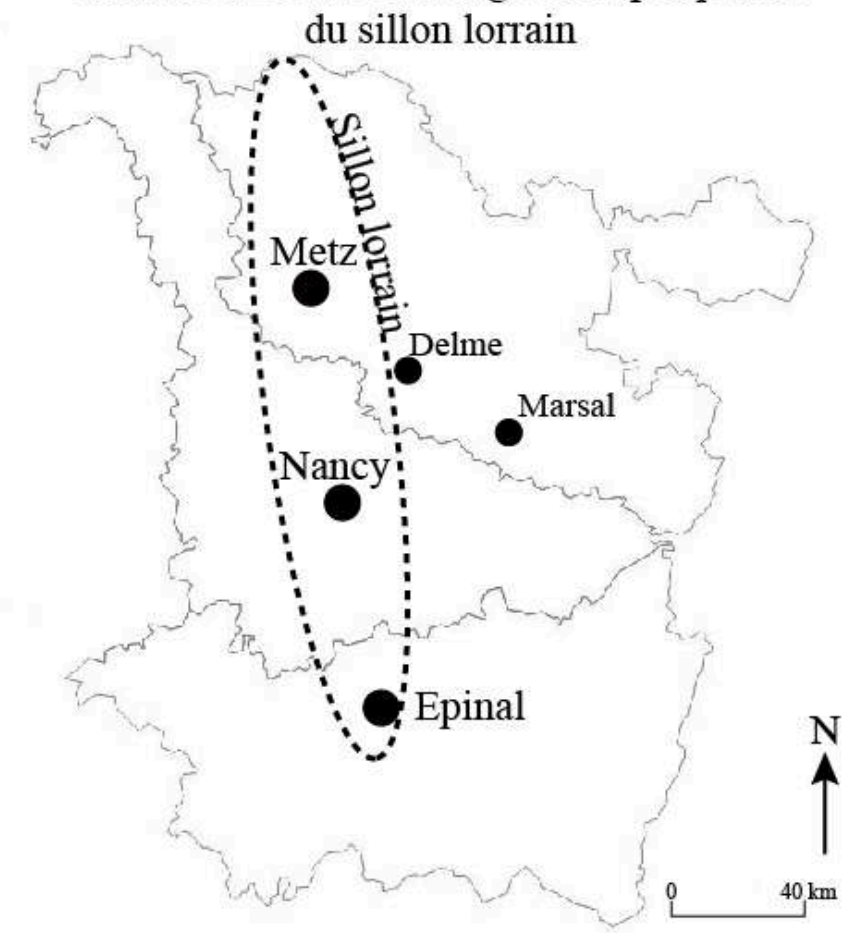

Conception : Gille, 2013

15 Les deux communes font parties de l'Est mosellan caractérisé par des faibles densités de population. Elles s'inscrivent dans le monde rural. Cette partie de la Moselle est également peu développée économiquement. 
Figure 3 : Delme et Marsal se situent dans le rural profond mosellan

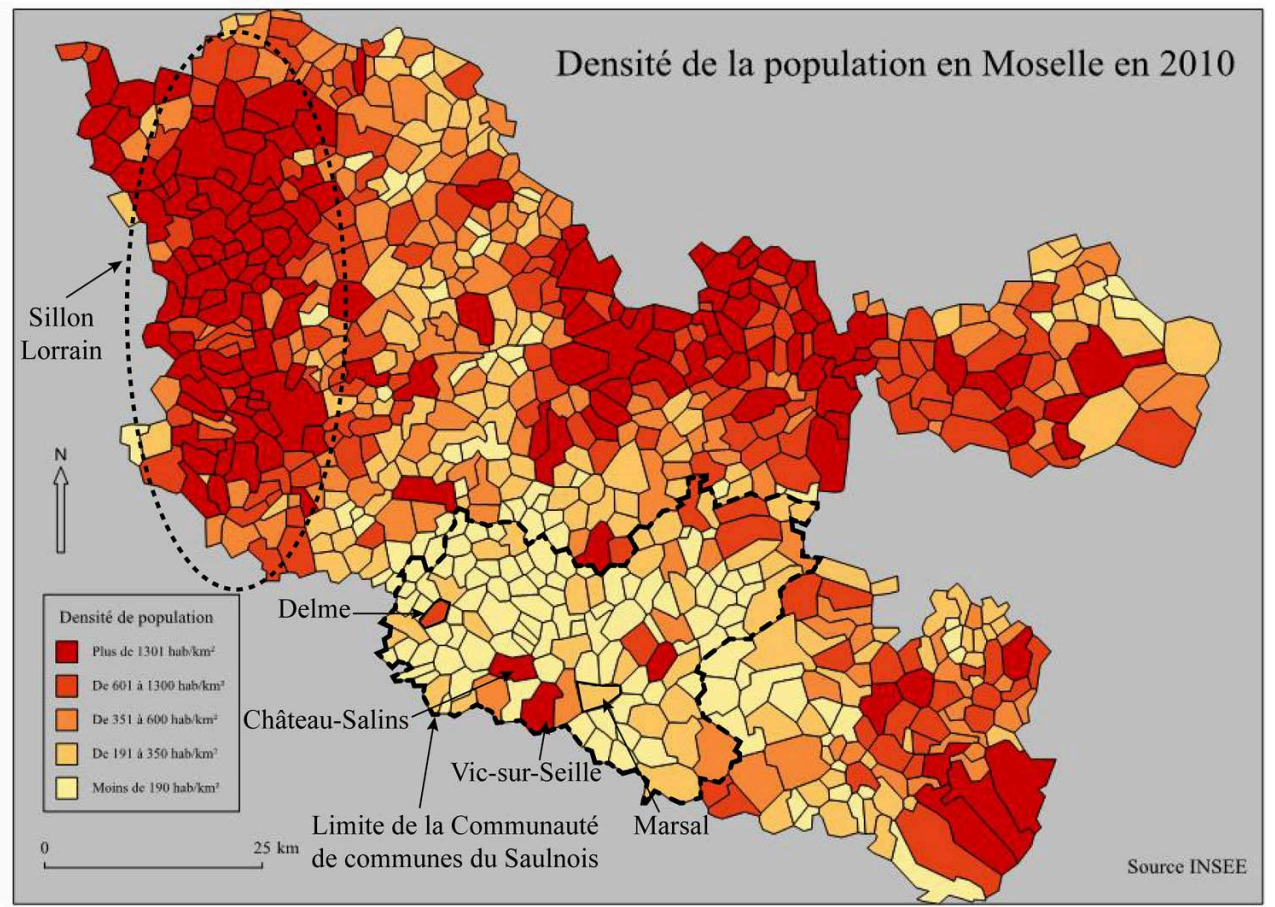

Conception : Gille, 2015

16 Eloignée des deux grandes villes lorraines et sans véritable rivale à proximité (ChâteauSalins reste suffisamment éloigné pour ne pas empêcher le développement démographique de Delme), Delme s'accroche à son statut de bourg centre (1052 habitants en 2012) si souvent malmené. Sa fonction de chef-lieu de canton, à l'intérieur de l'arrondissement de Château-Salins, renforce sa position et explique sa croissance toute relative grâce aux effets de la rurbanisation en marge du Sillon Lorrain. Delme est un cas à part en milieu rural, compte tenu de sa forte croissance démographique. Depuis quarante trois ans, la population croît d'environ 59\%. Cette évolution est à l'inverse de ce qui se rencontre en maints endroits. Face à une certaine inertie démographique dans l'arrondissement (diminution de 1,7\%), Delme fait figure de contre-exemple.

Tableau 1 : Une évolution variée de la population de Delme et de l'arrondissement de 1968 à $2012^{2}$

\begin{tabular}{|c|c|c|c|c|}
\hline Années & 1968 & 1975 & 1982 & 1990 \\
\hline Commune de Delme & 631 & 620 & 698 & 681 \\
\hline Arrondissement de Châteaul-Salins & 30465 & 29275 & 28739 & 28315 \\
\hline
\end{tabular}

17 Des activités tertiaires y sont présentes. Le ban communal est essentiellement occupé par des terres agricoles. 
Figure 4 : L'occupation du territoire de Delme

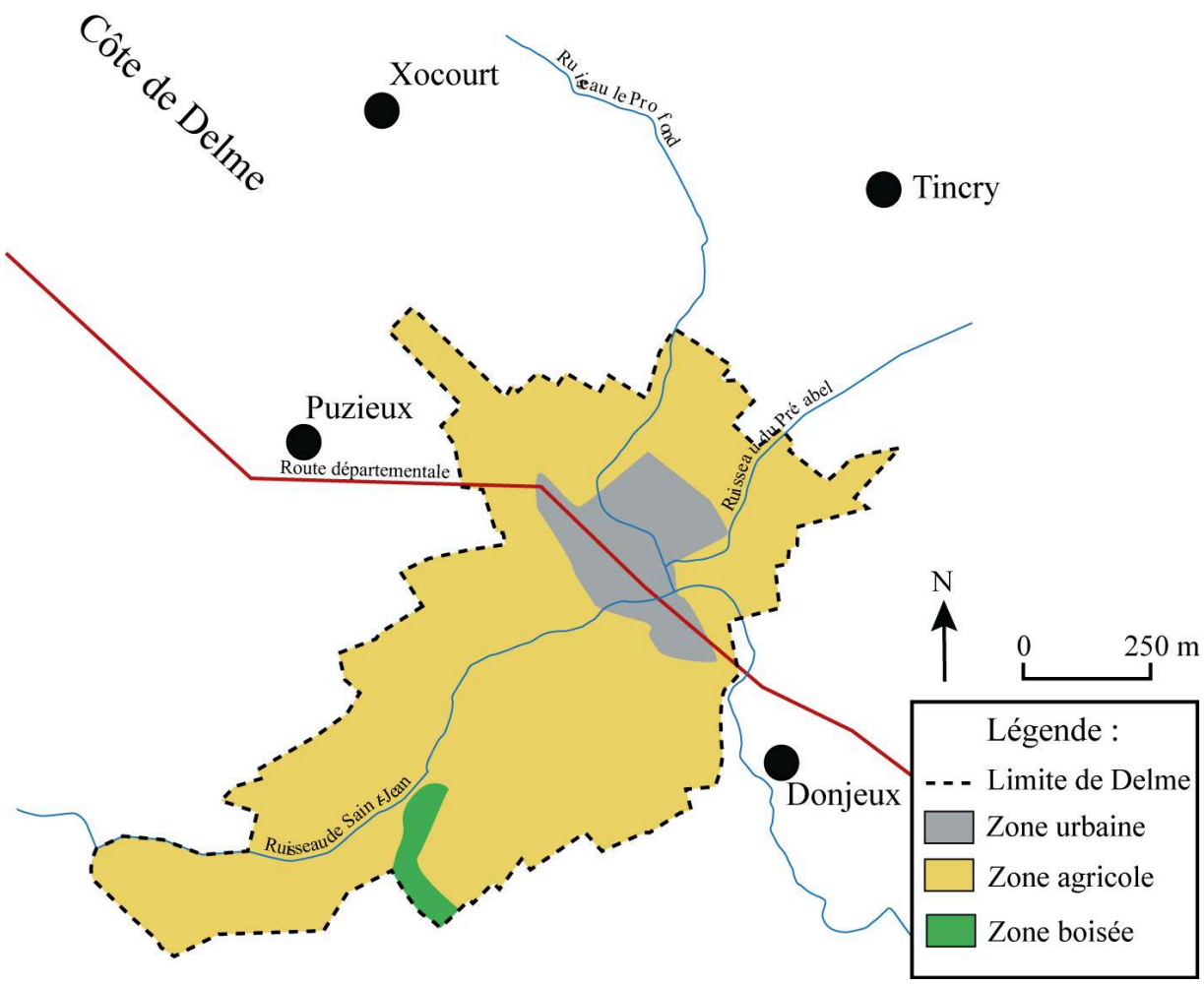

Conception : Gille, 2013

Le bourg est connu pour sa synagogue classée devenue lieu d'exposition. La poussée démographique de Delme n'est pas sans poser des problèmes environnementaux, sociaux et économiques. Les terres agricoles ont diminué de surface avec l'étalement du bâti. Les équipements publics doivent être adaptés aux changements démographiques : croissance, rajeunissement, nouvelles exigences.

La croissance démographique n'a pas toujours été anticipée. Elle est donc menée sans véritable politique de développement et de conservation du patrimoine. La mise en pratique d'un aménagement local durable peut être une clef d'entrée pour l'avenir. Marsal offre une situation différente. Bien que peu éloigné de Delme, la population a diminué de $10 \%$ en quarante trois ans atteignant seulement 252 habitants en 2012. Le minimum démographique de Marsal est actuel (tableau 2).

Tableau 2 : Une baisse de la population de Marsal et de son arrondissement

\begin{tabular}{|c|c|c|c|c|}
\hline Années & 1968 & 1975 & 1982 & 1990 \\
\hline Commune de Marsal & 301 & 287 & 280 & 284 \\
\hline Arrondissement de Châteaul-Salins & 30465 & 29275 & 28739 & 28315 \\
\hline
\end{tabular}

La commune, située à 30 kilomètres de la Porte Verte (zone commerciale) qui ouvre sur l'Est de l'agglomération de Nancy, reste pénalisée par la rugosité des distances-temps sur des voiries où la vitesse reste toujours limitée. Etabli dans la vallée de la Seille, le village reste encerclé par la rivière et ses marais. Seul le site initial du briquetage est situé sur une terrasse anthropique (comme le montre la figure 5 avec la carte des Naudins). 
Figure 5 : Le village de Marsal sur la carte des Naudins ${ }^{4}$

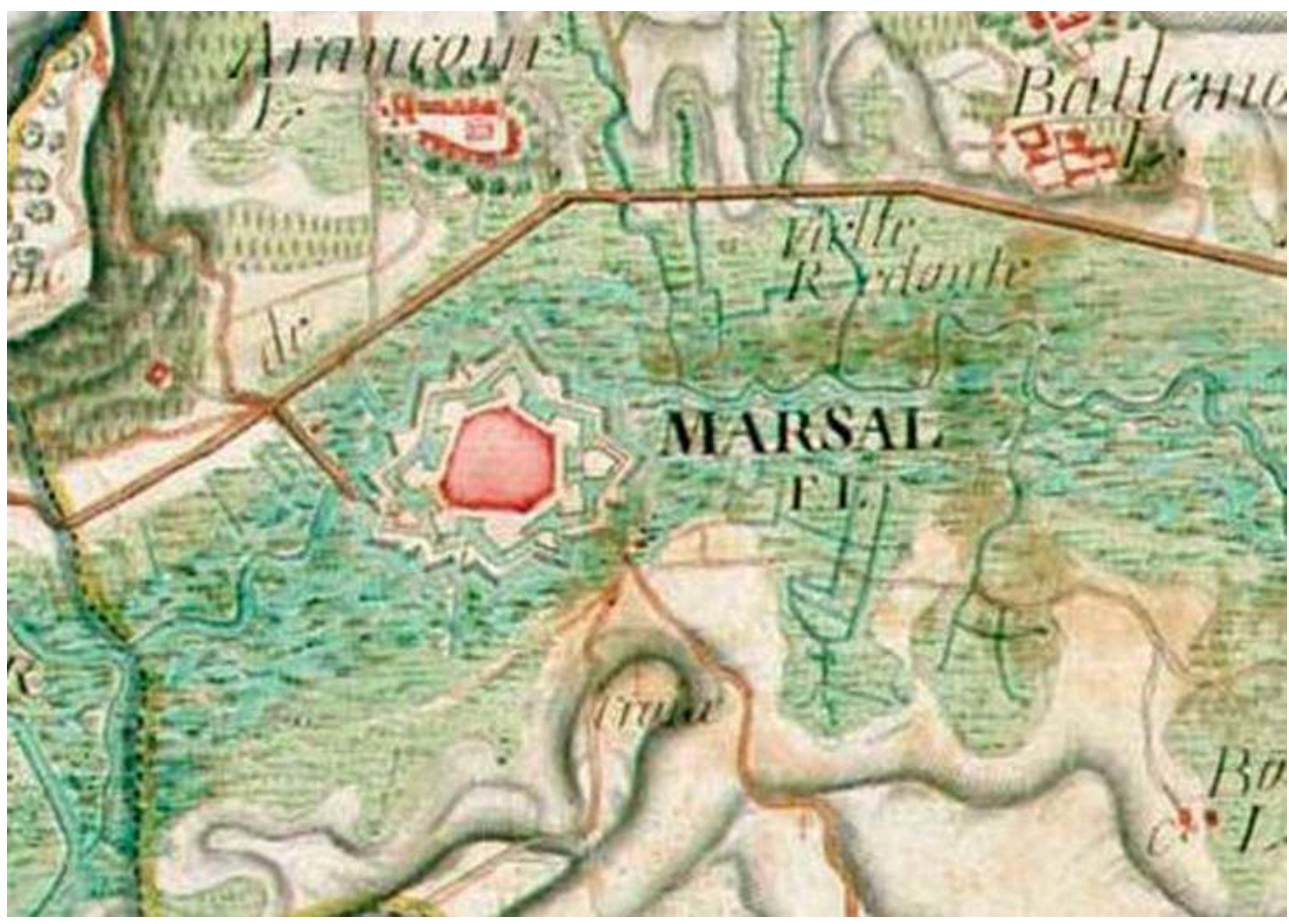

La terrasse anthropique est actuellement occupée par le village (de couleur rouge). Le village se situe au cœur de la vallée de la Seille.

Le potentiel local peut cependant permettre à Marsal de rebondir grâce à son patrimoine, élément souvent utilisé pour élaborer des projets de territoire (Landel, Senil, 2009).

Marsal est inclus dans le Parc Naturel Régional de Lorraine. La biodiversité locale exceptionnelle est riche grâce aux prés salés. Le passé historique est prestigieux. La prise de la ville par Louis XIV (1663) a été marquée par la versification de Jean de La fontaine en 1671. Marsal fut une place forte essentielle, imposée par Louis XIV, sur le chemin de l'Allemagne (Cabourdin, 1991).

Pour résumer, la réflexion porte sur plusieurs applications du développement durable dans l'aménagement rural. Delme est une commune rurale en développement à proximité du Sillon Lorrain mais sans réel attrait. Sans véritable enjeu particulier en matière d'aménagement du territoire, comment Delme parvient-elle à appliquer le développement durable dans ses projets? Marsal est commune rurale atrophiée, riche en patrimoine, dans un espace peu organisé. Comment la commune applique-t-elle le développement durable dans son aménagement sachant qu'il existe de nombreux documents de protection contraignants?

Avant d'étudier les deux communes, il faut au préalable fournir quelques éclairages concernant le développement durable et sa transposition dans la législation française. 


\section{Le développement durable, un concept international décliné localement}

Ce concept apparaît au niveau international pour s'imposer progressivement dans les législations nationales à l'image de la France.

\section{A. La naissance et la propagation du concept}

L'environnement est au cœur des premières actions internationales. Les effets des révolutions industrielles aboutissent aux premières conférences sur la protection de la nature et de la faune à la fin du XIXème siècle. En 1972, le Club de Rome alerte le monde avec la publication d'un rapport annonçant un épuisement des réserves mondiales de pétrole et de gaz dans les années 1990. Il exhorte les pays développés à repenser leurs modes de fonctionnement et dénonce les actions anthropiques sur la nature (Mancébo, 2012). La même année, la conférence de Stockholm initie la naissance du Programme des Nations Unis et du droit environnemental international.

Les années 1980 sont un véritable tournant. La notion d'écodéveloppement voit le jour. Ignacy Sachs (1980), le définit comme «le développement des populations par ellesmêmes, utilisant au mieux les ressources naturelles, s'adaptant à un environnement qu'elles transforment sans le détruire " (Ducroux, 2002). La même année, le concept de développement durable apparaît pour la première fois dans un document produit par le Programme des Nations Unies pour l'Environnement, l'Union Internationale pour la Conservation de la Nature et le Fonds mondial pour la vie sauvage. Il s'intitule « La stratégie mondiale de la conservation" (UICN, 1980). Le rapport Brundtland de 1987 marque officiellement le point de départ du développement durable. Établi à la demande des Nations-Unies, il préconise une bonne gestion des ressources naturelles et une économie de l'énergie compatible avec le potentiel des écosystèmes de la planète. Ce nouveau concept est consécutif au Global Change où les changements climatiques commencent à intéresser la communauté scientifique et internationale.

L'avènement du développement durable est contemporain, probablement lié à la fin de la guerre froide. Le développement, proposé après la seconde guerre mondiale, ne comporte plus aucun intérêt idéologique (Brunel, 2004). Le sommet de Rio, en 1992, arrive au bon moment en proposant un nouveau mode de développement. Il devient le fondement de la coopération internationale.

Dix ans plus tard, le sommet de Johannesburg fait le point sur les avancées prises en matière de développement durable. Ce sommet montre certaines limites (remise en cause des objectifs signés à Rio). La conception du développement durable diffère entre les pays industriels et ceux en voie de développement. Pour les premiers, le développement durable s'intéresse plus à la durabilité qu'au développement. Les seconds inversent les priorités et s'intéressent plus au développement qu'à la durabilité afin de permettre à leurs populations de sortir de leur condition de vie difficile (Veyret, 2004). Le développement durable se décline dans l'aménagement français. 


\section{B. La déclinaison française du développement durable}

31 La mise en place de l'arsenal législatif français débute en 1995. La loi Barnier renforce la protection de l'environnement et donne la première définition française du développement durable (Lorach et De Quatrebarbes, 2003). La loi fait du principe de précaution un élément du contrôle de légalité. Elle crée aussi le " principe de pollueur/ payeur » et le principe de participation et de transparence de l'information (chacun doit avoir accès aux données relatives à l'environnement).

Les lois Voynet et Chevènement de 1999 complètent le dispositif législatif. La loi Voynet crée deux nouvelles entités territoriales (le " pays » et "l'agglomération ») alors que la loi Chevènement crée la Communauté de communes, la Communauté d'agglomération et la Communauté urbaine. Elle est complétée en 2010 par une réforme des collectivités territoriales et crée de nouveaux groupements : la métropole, le pôle métropolitain et la commune nouvelle (Dantonel-Cor, 2011). Ces nouvelles collectivités doivent faciliter, en partie, l'harmonisation des politiques d'aménagement durable sur des territoires de grandes tailles.

33 En 2000, la loi Solidarité et Renouvellement Urbain (SRU) fournit un outil de gestion foncière en créant le Plan Local d'Urbanisme (PLU) et le Schéma de Cohérence Territoriale $(\mathrm{SCOT})$ généralisable à l'ensemble du territoire en 2017. Le Projet d'Aménagement et de Développement Durable (PADD), issu de la loi Voynet, est retranscrit dans le PLU et dans le SCoT.

34 En 2002 est votée la loi démocratie de proximité qui renforce la démocratie participative. Elle met en œuvre le principe de participation issu de Rio (Priet, Jacquot, 2008). Elle permet à la société civile d'être associée au processus d'élaboration des projets qui ont une incidence sur l'environnement ou l'aménagement du territoire.

Le Grenelle de l'environnement, et les lois qui en découlent, propose des mesures qui touchent les secteurs de l'énergie (d'éviter que les règles d'urbanisme ne gênent la mise en œuvre de travaux d'amélioration de la performance énergétique des bâtiments) et du bâtiment, des transports (privilégier les transports e commun), de la biodiversité (création de trames vertes et bleues), de la gouvernance et des risques pour l'environnement et la santé (Graindorge, 2001).

36 La réforme territoriale de 2010 arrête la création des " pays » (Tautelle, 2010). Enfin, la loi pour l'Accès au Logement et un Urbanisme Rénové (ALUR) de 2014 contraint à « engager la transition écologique des territoires" (en privilégiant la densification au détriment de la consommation des espaces) et à faire évoluer les règles d'urbanisme pour proposer un aménagement respectant mieux la durabilité (en incitant à élaborer uniquement des PLU au niveau des intercommunalités).

37 L'Etat crée donc les outils nécessaires et contraignants pour mettre en œuvre le développement durable (Cormier, De Lajarte, Carcaud, 2010 ; Theys, 2002). Une des premières mesures de l'Etat, en milieu rural, est la création des Parcs naturels régionaux en 1983. Leurs missions consistent à protéger le patrimoine naturel et culturel des communes membres ${ }^{5}$. Sa réussite passe par les acteurs locaux de nos territoires. 


\section{Des incitations multi-échelle au développement durable en milieu rural}

Au niveau local, et à partir des deux sites retenus (Delme, Marsal), interrogeons-nous les acteurs du territoire durable pour connaître leurs interventions sur des territoires imbriqués dans des échelles complémentaires et hiérarchisées (Plottu E, Plottu, B, 2009). ruraux. Le Groupe d'Action Local (GAL) Moselle Sud est chargé de le mettre en œuvre sur l'arrondissement de Château-Salins. Ses missions visent à identifier et accompagner les porteurs de projet. Delme et Marsal sont particulièrement concernés. La stratégie du programme Leader Moselle Sud vise à renforcer l'attractivité et affirmer la vocation touristique du territoire. Aider à faire connaître les mares salées est un exemple pour promouvoir la nature tout en valorisant l'attrait touristique du site.

Figure 6 : Schéma des politiques européennes

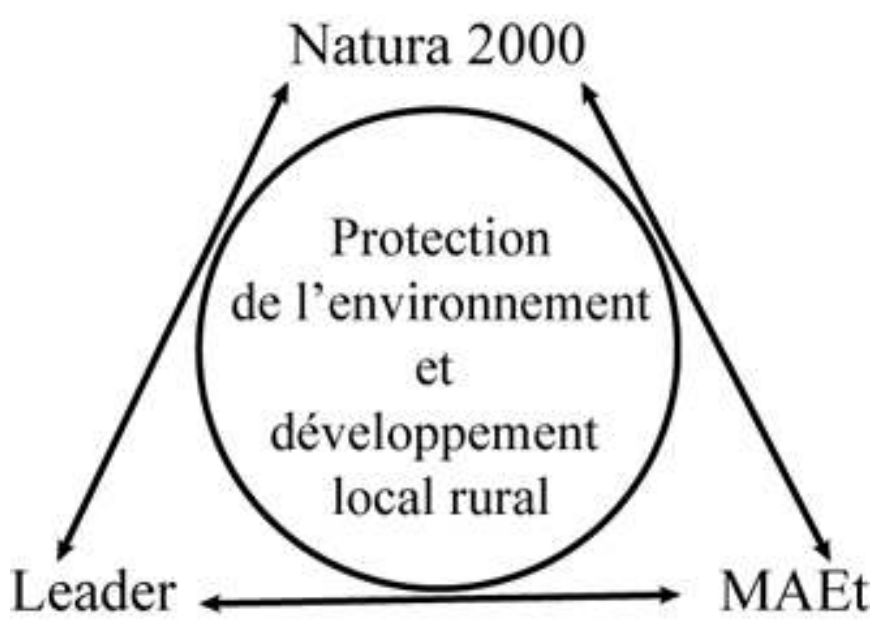

Conception : Gille, 2013

- L'Etat à travers ses services déconcentrés (Direction Départementale des Territoires (DDT), Direction Régionale de l'Environnement, de l'Aménagement et du Logement (DREAL), la Direction Régionale des Affaires Culturelles (DRAC)) :

En plus de fournir le cadre juridique pour aider les communes rurales à se développer durablement, l'Etat à travers la déconcentration ("processus d'aménagement de l'État unitaire qui consiste à implanter dans des circonscriptions locales administratives des autorités administratives représentant l'État ») assiste les territoires ruraux en participant à leurs projets.

44 La DDT offre un appuie juridique à la réalisation des documents d'urbanisme (pour Delme et Marsal). L'ancienne Direction Départementale de l'Agriculture et de la Forêt 
(DDAF) fait aujourd'hui partie de la DDT. Ce service participe aux documents d'urbanisme lorsque les communes possèdent de la forêt sur leurs territoires.

La DREAL pilote les politiques de développement durable issues des lois Grenelle. Depuis 2010, les différentes DREAL ont remplacé les DIREN (Direction Régionale de l'Environnement), les DRE (Direction Régionale de l'Équipement) et les DRIRE (Direction Régionale de l'Industrie, de la Recherche et de l'Environnement).

Le Service Territorial de l'Architecture et du Patrimoine (STAP) fait partie de la DRAC qui exerce une fonction de conseil auprès des collectivités locales. La DRAC fut associée à l'élaboration de la carte communale de Marsal compte tenu des différents sites classés aux Monuments Historiques qui existent (dont la citadelle fortifiée par Vauban). Sa mission fut de conseiller la commune afin d'élaborer un développement (création d'un nouveau lotissement en cœur de village) ne remettant pas en cause la qualité architecturale de l'ensemble.

47 - La Région (Lorraine) :

48 Le Schéma Régional d'Aménagement et de Développement Durable du Territoire (SRADDT) définit les objectifs relatifs à une localisation plus cohérente des grands équipements et des infrastructures. Il ne possède pas de valeur normative. La prise en compte du document dans les politiques d'aménagement relève du choix des collectivités. Cette absence d'opposabilité constitue une insuffisance.

Depuis 2009, le Schéma Régional de Cohérence Écologique (SRCE) participe à la préservation de l'environnement. Il s'élabore conjointement avec la Région, l'État, les EPCI (Établissements Publics de Coopération Intercommunale) compétents en matière d'aménagement de l'espace et les PNR. Les documents de planification, les projets des collectivités territoriales et de l'Etat doivent prendre en compte ce nouvel outil. En 2012, la région débute la réalisation d'un SRCE. Le SRCE devrait entrer en vigueur fin 2015.

50 - Le Département (de la Moselle) :

51 Le Conseil Général participe à la réalisation d'une approche durable du territoire par l'intermédiaire du Programme d'Aide aux Communes et aux Territoires Mosellans (PACTE). La démarche est partenariale. Elle est établie entre le département, les acteurs publics locaux et les chambres consulaires. Ils définissent ensemble une stratégie d'aménagement et de développement durable du territoire mosellan. PACTE constitue l'outil du département pour continuer l'effort d'aménagement durable des territoires de la Moselle. Le programme se décline en trois volets (aménagement, environnement et patrimoine).

52 En 2013, Marsal en a bénéficié dans le cadre d'un financement pour la construction d'une nouvelle passerelle pour l'aménagement de la mare salée (volet environnement du PACTE). La commune a obtenu une subvention de base de $35 \%$. Mais comme ce projet est considéré comme prioritaire par le Conseil Général, Marsal a obtenu une subvention supplémentaire qui couvre $70 \%$ du coût total. Ce type de supplément tend à orienter les communes vers les objectifs durables du département. Le cas de Marsal fut donc école. Delme a bénéficié d'une subvention pour sa salle des fêtes. Cette équipe a pour vocation de renforcer lien social. Il s'agit d'un lieu de manifestation et d'un lieu de réunion pour les habitants (via des associations par exemples).

PACTE présente un inconvénient majeur. Les projets subventionnés nécessitent un investissement conséquent. Certaines petites communes rurales ne pouvaient se 
permettre d'engager un tel investissement. La généralisation des Communautés de communes devrait aider à lever ce handicap. l'exemple de la charte, la notion de projet prend de l'essor en milieu rural grâce aux procédures contractuelles (Perrier-Cornet, 2002). Elle est conçue de manière concertée avec les différentes échelles d'acteurs. Marsal fait partie du territoire du Parc. Lorsque le PNRL intervient sur la commune, il applique sa charte. Il axe certaines problématiques sur le développement durable. Lors des réunions d'élaboration de la carte communale, le chargé de mission du Parc a axé ses interventions sur le fait que la carte communale doit respecter la charte. La carte doit être en accord avec les cinq finalités du développement durable ${ }^{6}$. Son avis émis lors de la consultation des personnes publiques associées est obligatoirement pris en compte.

Figure 7 : La charte du PNRL et l'aménagement durable des territoires

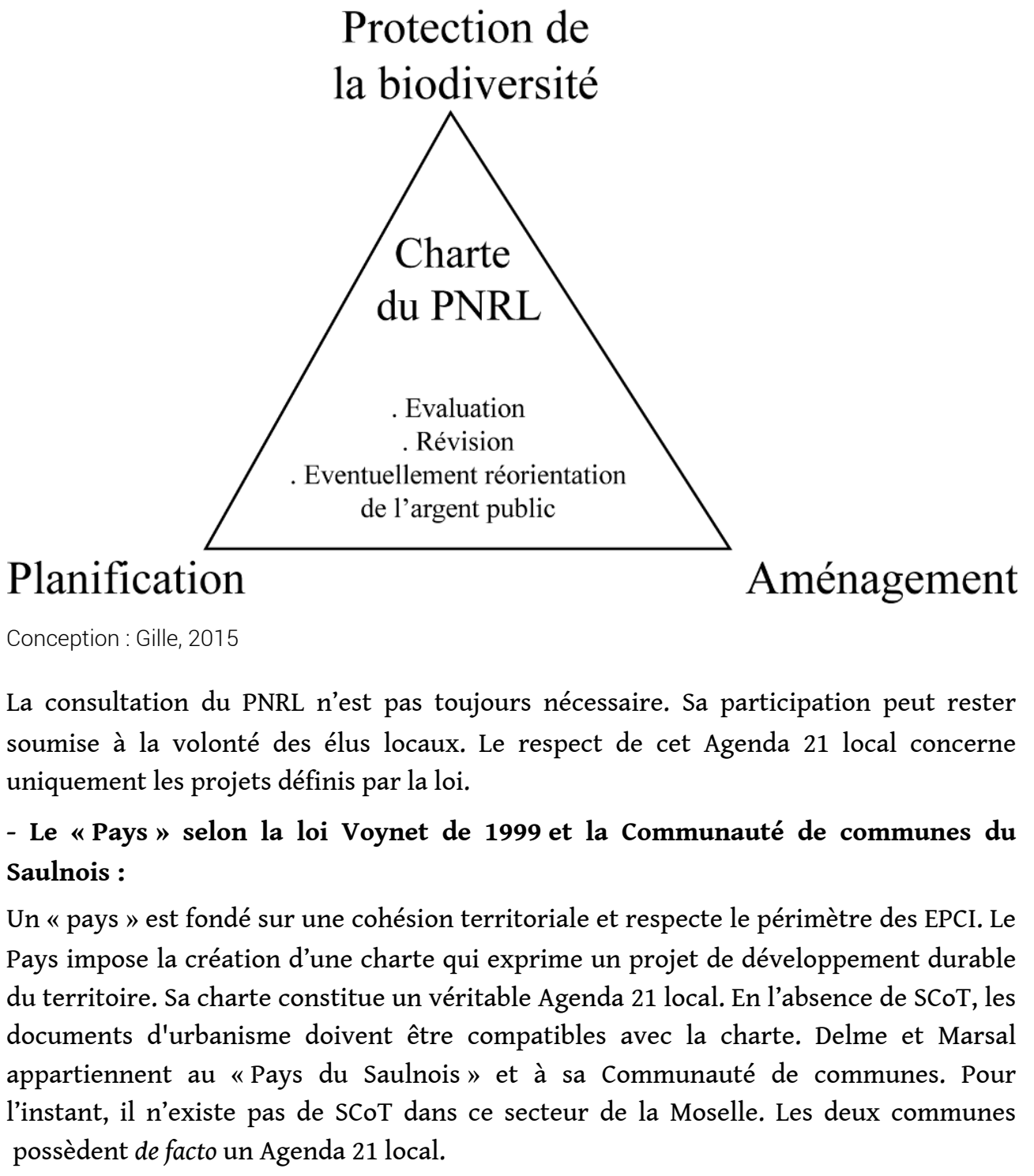

Protection de a biodiversite ntuellement réorientation l'argent public

\section{Planification}

Aménagement La consultation du PNRL n'est pas toujours nécessaire. Sa participation peut rester soumise à la volonté des élus locaux. Le respect de cet Agenda 21 local concerne uniquement les projets définis par la loi.

- Le « Pays» selon la loi Voynet de 1999 et la Communauté de communes du Saulnois :

Un « pays » est fondé sur une cohésion territoriale et respecte le périmètre des EPCI. Le Pays impose la création d'une charte qui exprime un projet de développement durable du territoire. Sa charte constitue un véritable Agenda 21 local. En l'absence de SCoT, les documents d'urbanisme doivent être compatibles avec la charte. Delme et Marsal appartiennent au "Pays du Saulnois" et à sa Communauté de communes. Pour possèdent de facto un Agenda 21 local. 
Un plan d'actions applique la charte composée d'axes stratégiques. Chaque axe se décline en volets d'orientation puis en actions concrètes. Un des volets vise à " structurer le territoire autour des bourgs centres et intermédiaires de Delme, Château-Salins, Dieuze, Vic-sur-Seille ». Une des actions consiste à étudier les possibilités de déviation de Delme par la mise en place d'une voie de contournement (figure 3). Lors de la constitution du PLU de 2003, nous constatons dans le zonage que les parcelles situées au sud du village sont classées en zone Agricole (A) et non en zone A Urbaniser (AU) pour permettre le contournement. Les élus ont anticipé la préconisation de la charte en y intégrant cette donnée.

Figure 8 : L'adaptation du zonage de 2003 de Delme à la charte de Pays

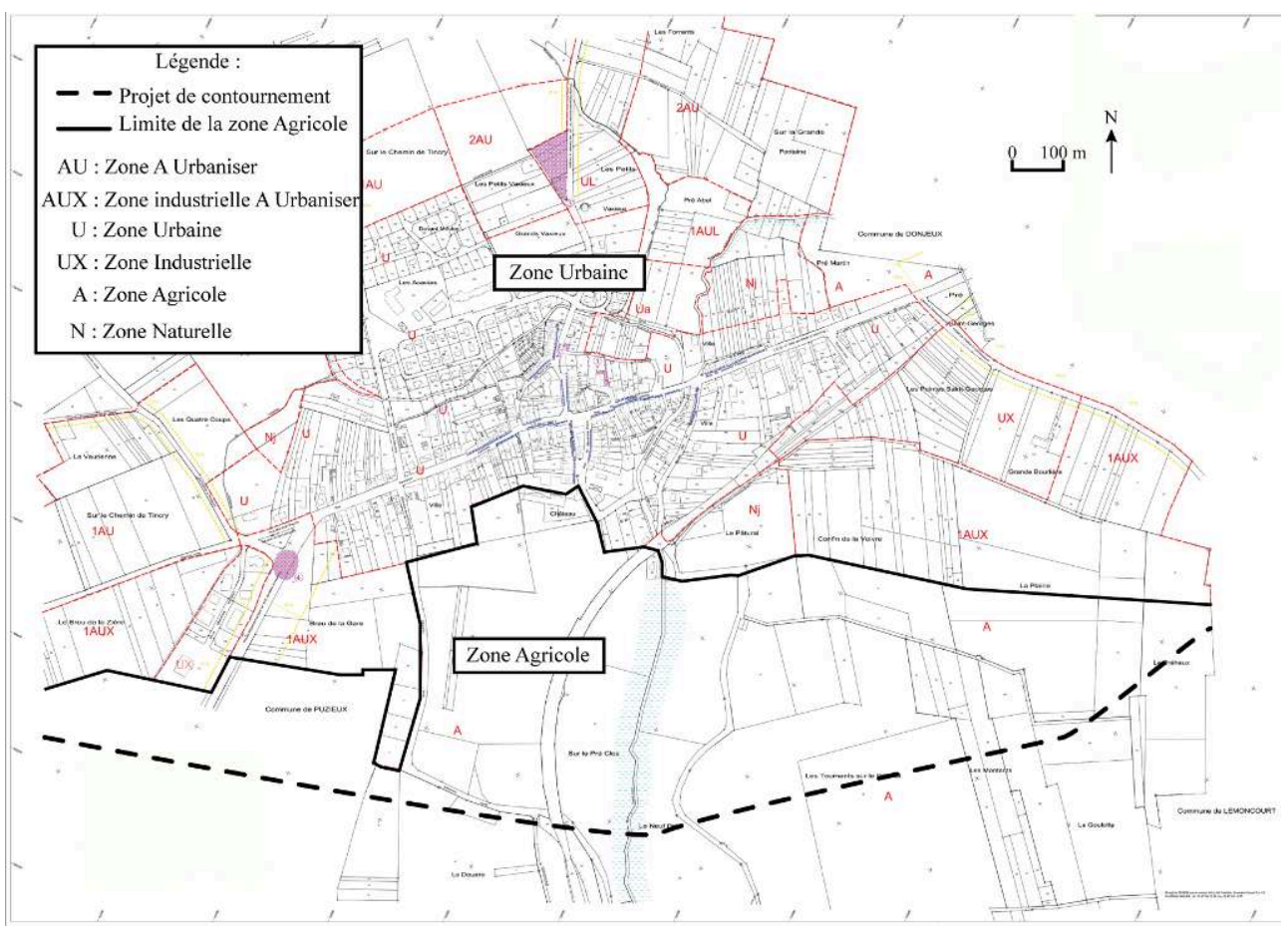

Conception : Gille, 2013

Le contournement diminue le trafic routier à l'intérieur du village et favorise la sécurité aux abords des voies routières. Malheureusement, le projet présente un inconvénient de taille. Le contournement nécessite la destruction de terrains agricoles essentiellement occupés par des champs voués à la culture céréalière. Ces terrains sont potentiellement traversés par la faune locale. Bien que le projet existe dans la charte du Pays qui constitue un Agenda 21 local, le contournement n'est pas un projet d'aménagement durable.

61 La Communauté de communes accueille le Pays du Saulnois. Elle l'a absorbé, ce qui crée une situation originale car l'EPCI gère les projets du " pays». Cette situation n'est pas rare en France car les EPCI ont tendance à prendre le pilotage des projets de territoire au détriment des « pays » (Gilly, Wallet, 2005).

- Les autres organismes de consultation :

La chambre d'agriculture de Moselle a participé aux réunions de travail de la carte communale de Marsal. Sa mission consiste à défendre les intérêts des agriculteurs toujours à la recherche de SAU. 
64 Le Conseil d'Architecture, d'Urbanisme et de l'Environnement (CAUE) est une association départementale qui conseille les particuliers et les collectivités dans les domaines de l'architecture, de l'urbanisme et de l'environnement. Les communes peuvent adhérer à l'association qui apporte son expérience dans le cadre des documents d'urbanisme. Le CAUE de la Moselle n'est pas intervenu sur nos deux territoires. Leur avis est obligatoirement pris en compte lors de l'élaboration des documents d'urbanisme. L'aménagement rural durable s'exprime de différentes manières.

\section{Les politiques d'aménagement durable prennent une place de plus en plus importante en milieu rural}

65 Les études de cas des communes de Delme et Marsal le confirment. Le développement durable en milieu rural est présent à la fois dans les documents d'aménagement ayant une portée juridique mais aussi dans les simples projets de développement.

\section{A. La planification rurale durable passe essentiellement par les documents d'urbanisme}

Les deux communes ont fait l'expérience de plusieurs cas d'application du développement durable dans leur document d'urbanisme.

\section{1) La carte communale de Marsal}

Le diagnostic paysager permet de justifier les choix d'orientations d'aménagement des élus et d'aborder les réalisations concrètes.

\section{a) Diagnostic paysager}

Sur Marsal, les maisons s'entassent sur un espace restreint encerclées par les anciens remparts. La commune conserve la typologie d'une ancienne ville. Elle possède de nombreuses parcelles occupées par des jardins qui représentent une surface susceptible d'être urbanisée. 
Photographie 1 : Vue aérienne oblique ouest-sud-ouest du village de Marsal dans son environnement ${ }^{7}$

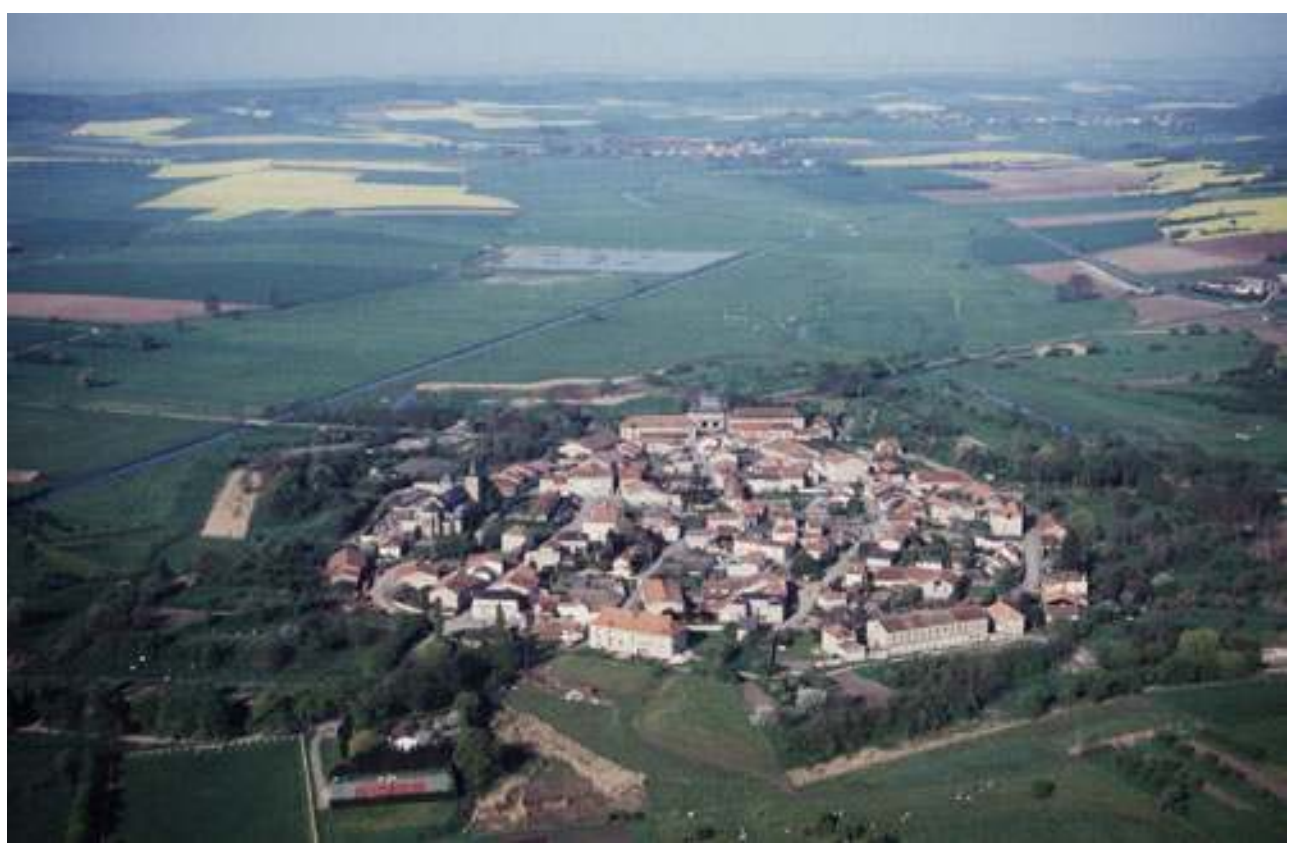

Cette photographie aérienne montre la forme ancienne et actuelle du village. L'habitat est concentré à l'intérieur des anciennes fortifications occupées par la végétation. Les prairies et la Seille entourent le village. La zone agricole est ensuite présente.

69 La partie sud et la partie ouest du village rassemblent un bâti urbain dense. La place d'Armes correspond au centre du village. La plupart des institutions du village s'y retrouvent : mairie, école et église. Dans ces trois parties, le bâti laisse place à un alignement plus ou moins strict de maisons et d'anciennes fermes.

Dans la partie nord-est du village, le bâti est beaucoup moins dense. Il laisse place à de nombreuses maisons individuelles non mitoyennes qui reflètent plusieurs époques de construction contemporaines. Aujourd'hui, le bâti est beaucoup moins dense.

71 Une autre forme urbaine vient s'ajouter. Il existe un lotissement à l'extérieur du village. Il est constitué d'un alignement de maisons individuelles sur un seul côté de la voirie.

72 L'ensemble du village comporte des nombreuses « dents creuses ». Il s'agit de parcelles non bâties. Elles sont utilisées comme vergers ou jardins. Cela donne un caractère urbain atypique. Les jardins ne sont pas exclusivement situés à l'arrière des maisons. Ils sont présents de manière inégale en bordure de voie et sont à la vue de tous. 


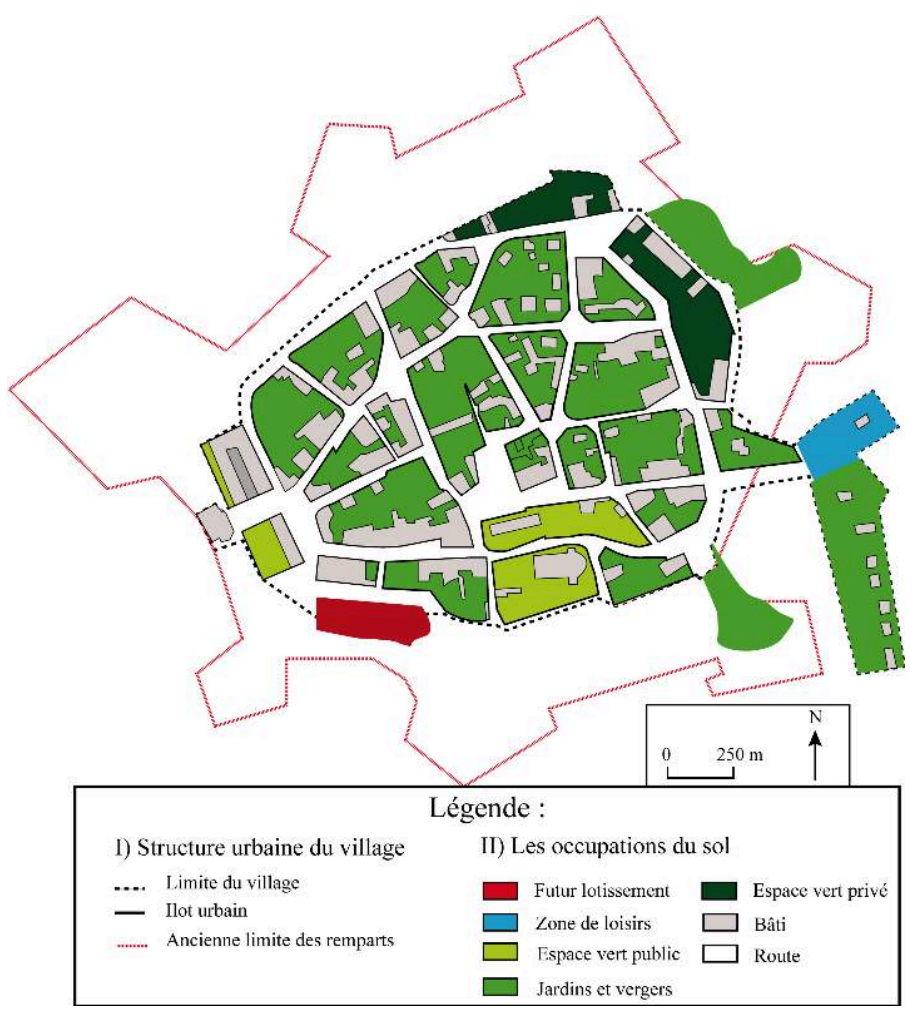

Conception : Gille, 2013

73 Les réseaux nécessaires existent déjà dans la mesure où beaucoup de jardins sont en fait des dents creuses. La carte communale traduit les choix du conseil municipal.

\section{b) Présentation et analyse des orientations envisagées dans le document d'urbanisme}

L'évolution juridique de la carte communale oblige les communes à s'inscrire dans une optique durable (articles L 110 et L 121-1 du code de l'urbanisme). Ces articles ont connu une adaptation majeure avec les lois Grenelle. Le Règlement National d'Urbanisme $(\mathrm{RNU})^{8}$ participe lui aussi à cette prise en compte par les règles relatives à la construction.

75 A l'intérieur de la carte communale, le volet environnemental est présent dans le parti d'aménagement (il s'agit du projet de développement retenu par la commune). La figure 9 présente les objectifs retenus : densification du bâti à l'intérieur du village et préservation de la surface agricole utile (SAU) et des corridors biologiques en interdisant les implantations humaines là où elles s'opposent à cette logique de connexité.

Trois parcelles internes ont été aménagées depuis l'entrée en vigueur de la carte communale en 2009. Construire à l'intérieur du village participe à la préservation des espaces naturels atypiques et de la SAU. Les interdictions dictées par ce cadrage protègent les milieux naturels (mare salée, vergers) et les corridors biologiques (zones de repos des oiseaux, les zones humides, la mare salée). 
Figure 10 : Le parti d'aménagement densifie le bâti et préserve la SAU ${ }^{9}$

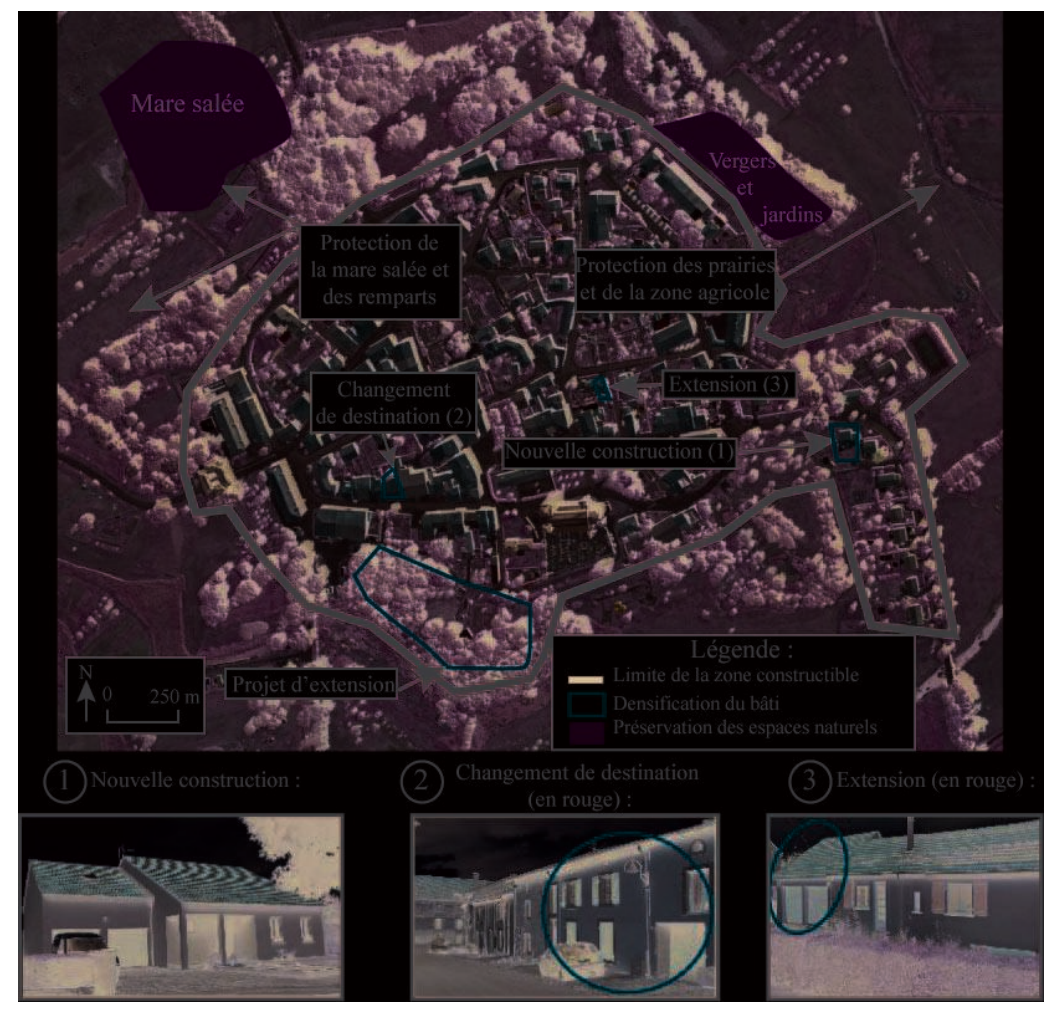

Conception : Gille, 2013

77 Le RNU impose un respect des préoccupations environnementales définies dans le code de l'environnement. L'installation de panneaux photovoltaïques participe à la concrétisation de ce respect. La mairie a accordé deux autorisations (figure 11). 
Figure 11 : L'installation de panneaux solaires ${ }^{10}$ dans le village de Marsal

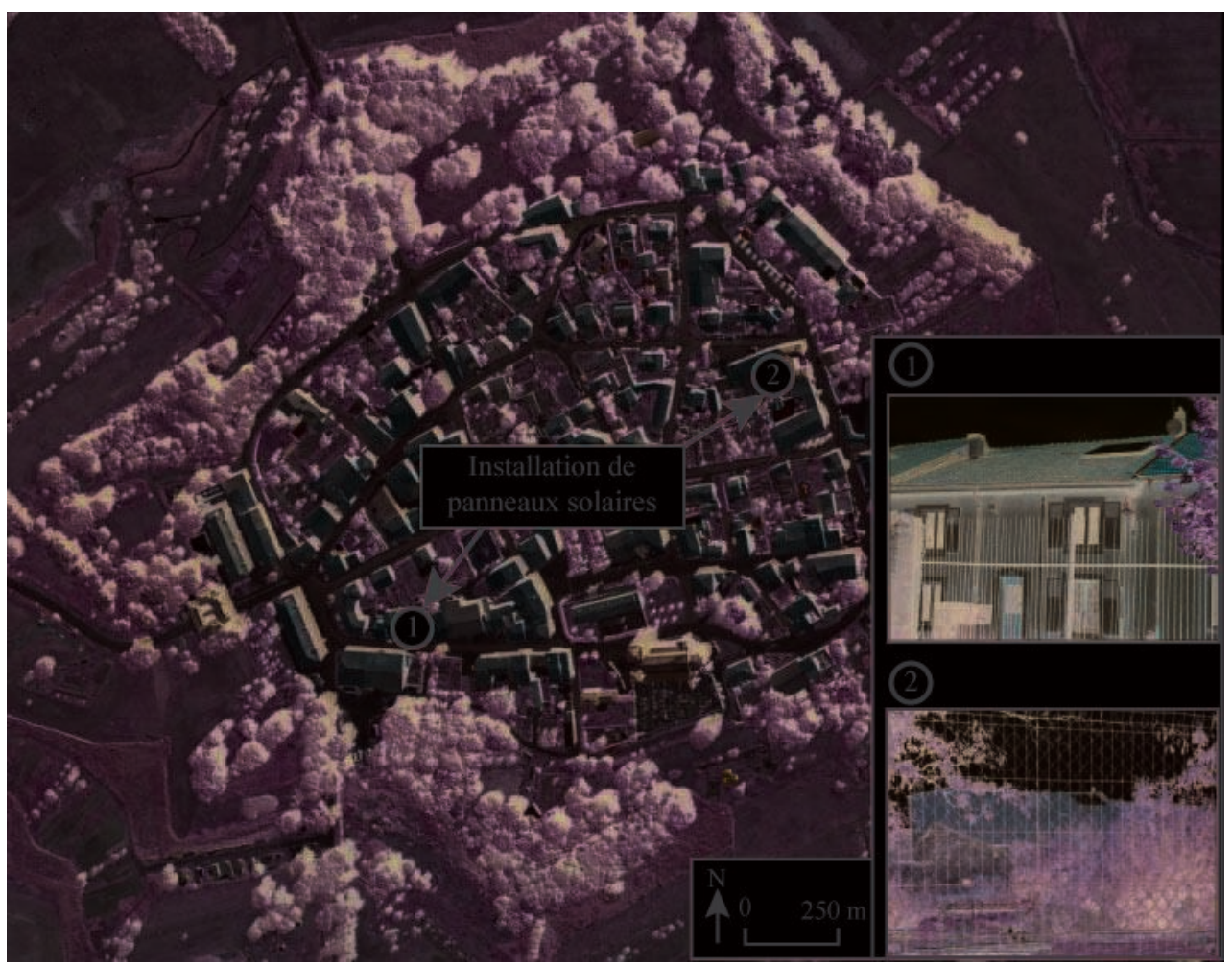

Conception : Gille, 2013

78 La prise en compte du volet social souhaite favoriser l'arrivée de nouveaux habitants. Il laisse la place à la création d'un lotissement toujours en projet. L'approche économique se contente de maintenir les activités commerciales existantes sans permettre la création de nouvelles. Le développement économique et social à travers la carte communale de Marsal reste limité. La carte communale a essentiellement pour mission de définir des zones constructibles ou non constructibles. A travers sa mission, elle agit principalement sur l'aspect environnemental. En fait, l'essor à attendre dépend surtout de l'évolution des distances-temps acceptées pour atteindre l'entrée de ville de Nancy appelé Porte Verte. Les orientations de Delme diffèrent de Marsal.

\section{2) Le PLU de Delme}

79 Le diagnostic paysager de Delme influence le développement communal.

\section{a) Diagnostic paysager}

80 Delme s'apparente à un village-rue. Ce type de village est le plus fréquent en Lorraine. Il est entouré de meix (dépendances attenantes à un jardin ou verger). Ils forment la zone tampon entre l'espace urbain et l'espace agricole. Delme laisse apparaître plusieurs spécificités : l'étroitesse de la parcelle bâti et la jointivité des façades des habitations en bordure d'usoir. 
Figure 12 : Vue aérienne oblique du village de Delme et son croquis d'interprétation

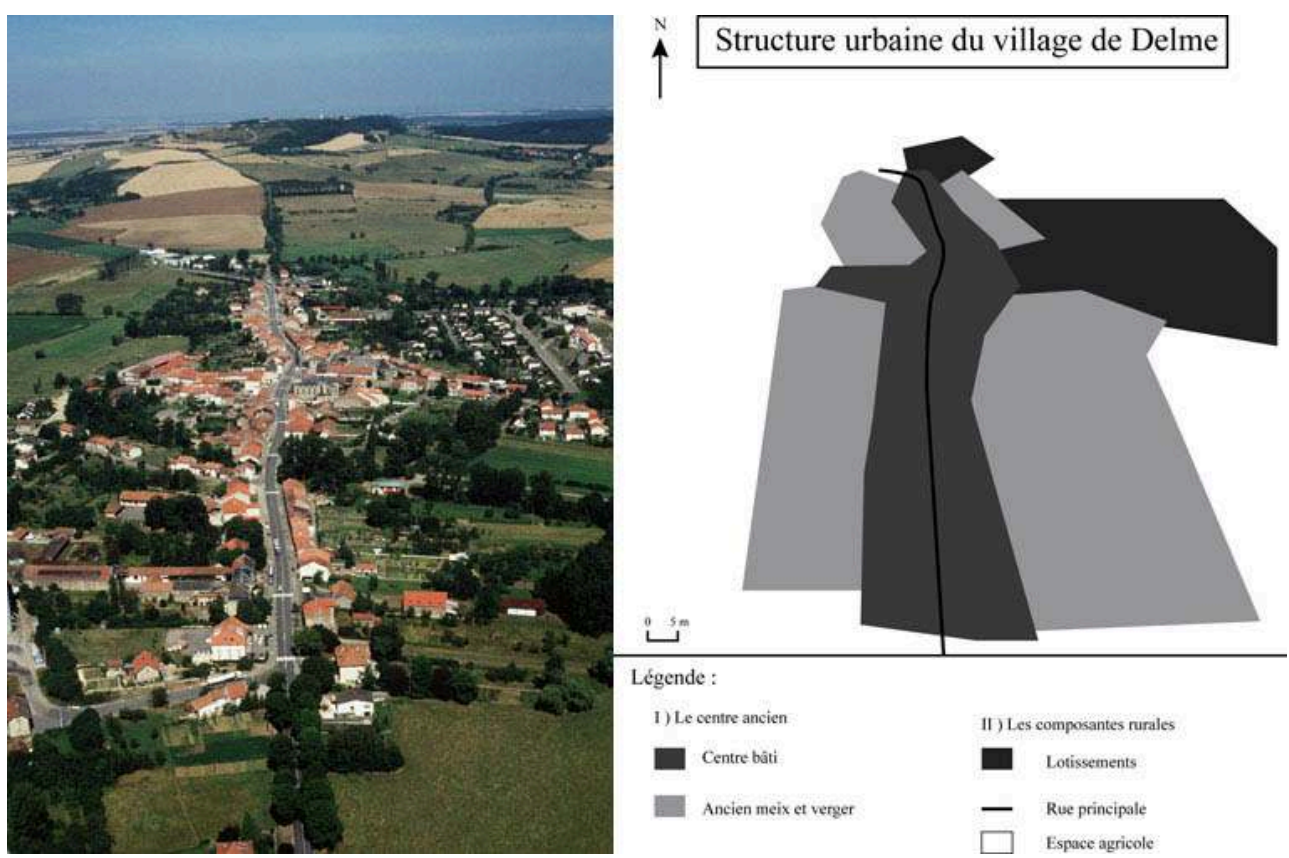

Conception : Gille, 2013. La vue aérienne oblique est orientée nord-ouest. Nous distinguons la rue principale bordée par des maisons. Derrière celles-ci, des anciens meix et vergers sont visibles. Le village s'est étendu avec la création de lotissements. Le schéma de synthèse permet d'identifier les différentes structures.

81 Les parcelles inoccupées ou «dents creuses » à l'intérieur du village traduisent la disparition de certaines bâtisses. Les habitations les plus récentes se situent en périphérie du village. Elles sont localisées dans la partie nord, sous forme de lotissements (maisons individuelles non jointives) (figure 12). Les zones industrielles sont installées prioritairement le long de la route départementale en entrée de village.

Le Plan Local d'Urbanisme présente les orientations de développement.

\section{b) Présentation et analyse des orientations envisagées dans le document d'urbanisme}

Les orientations d'aménagement, retenues dans le PADD (Projet d'Aménagement et de Développement Durable), préconisent d'éviter un étalement urbain. Elles favorisent une densification du bâti et veulent conserver intact le patrimoine architectural, naturel et culturel de la commune. Parvenir à maintenir, voire à développer l'emploi sur la commune est un autre objectif. Enfin, préserver la SAU s'impose. Qu'en est-il dans les faits? Tous les enjeux ne sont pas encore atteints. 
Figure 13 : La réalisation des objectifs du PADD de Delme ${ }^{11}$

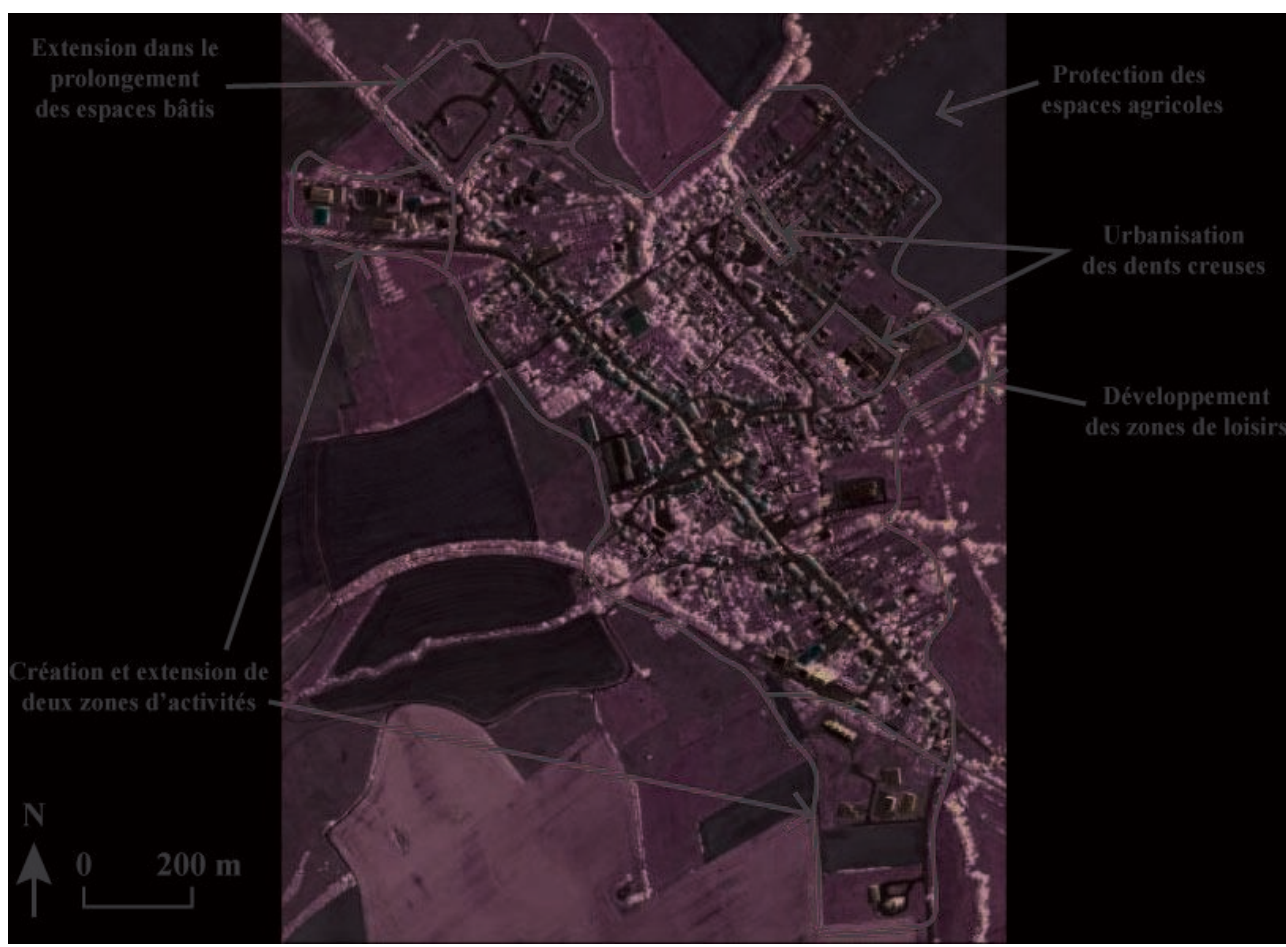

Conception : Gille, 2015 interdictions dictées. L'urbanisation des «dents creuses» est préconisée. L'extension dans le prolongement des espaces bâtis est réalisée par la création de lotissements. Les zones industrielles sont renforcées par leur extension. Les espaces agricoles restent préservés de toute urbanisation.

résultat doit être nuancé. Malgré les orientations du PADD, la zone agricole a été fortement réduite entre 1989 et 2003. Les zones agricoles et naturelles réunies diminuent de 83 à $71 \%$ au profit des zones à urbaniser (lotissements). Bien que le PADD mette en place des objectifs de développement durable, il reste difficile de réaliser les objectifs affichés dans le zonage. La diminution des zones agricoles et naturelles reste relative. Le fait de classer une parcelle en zone AU n'impose pas son utilisation immédiate.

Le PLU a été modifié pour s'adapter à l'évolution législative. Une première modification crée une zone naturelle au cœur de la zone urbaine pour préserver des parcelles occupées par des jardins et des anciens vergers. La trame verte et urbaine du village est ainsi maintenue. Les anciens meix sont protégés contrairement aux prescriptions du zonage de 2003 avec un classement des terrains en zone naturelle soit en terrain inconstructible.

87 La réglementation en faveur de la durabilité a évolué depuis l'élaboration du PLU. Le règlement $\mathrm{du}$ PLU autorise et encourage désormais l'utilisation de matériaux renouvelables. Son application est visible dans le paysage urbain.

88 Les modifications apportées au PLU de 2003 ne peuvent pas remplacer la réalisation d'un véritable PLU Grenelle. Les opérations d'aménagement revêtent un aspect durable. 


\section{B. Les opérations d'aménagement : des réalisations concrètes de projets de développement durable}

Le lotissement durable à Delme et la mare salée à Marsal correspondent à des exemples d'aménagements durables en milieu rural.

\section{1) Présentation et analyse du lotissement durable à Delme}

La commune a lancé la construction d'un lotissement durable en 2007. Ces projets se multiplient dans les campagnes. Ses caractéristiques visent à prévoir une gestion alternative des eaux pluviales avec un bassin de rétention équipé de roseaux, à favoriser l'encouragement des modes de déplacement doux, à orienter les constructions de façon à profiter au maximum du soleil, à réduire les ouvertures en façade nord pour limiter les déperditions thermiques. Malheureusement, la réalité est quelque fois différente. Certaines façades côté jardin sont orientées vers le nord. Une seule maison possède des panneaux photovoltaïques. Aucune toiture végétale n'existe bien que le règlement le propose. Le règlement limite aussi l'emprise au sol des constructions. Chaque parcelle possède une emprise végétale variant entre 35 à $50 \%$ de sa surface. $40 \%$ d'une unité foncière doit être traitée en espace vert.

Figure 14 : Les aménagements durables dans le lotissement « les terrasses d'Hélios ».

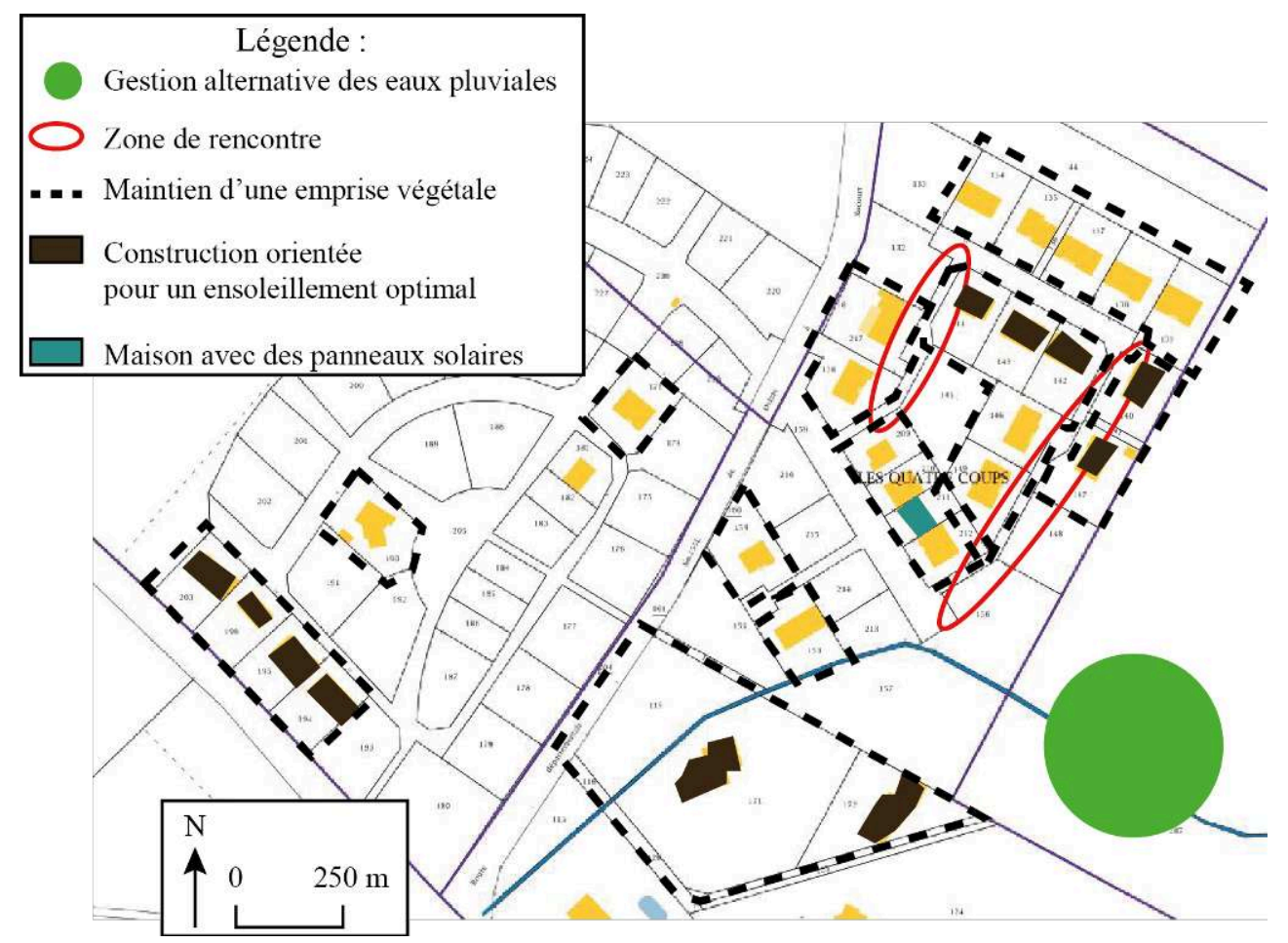

Conception : Gille, 2013

91 La durabilité du projet peut être remise en question. Dans l'esprit des acquéreurs, il est difficilement concevable de vivre, sur une commune rurale, dans un appartement. Le monde rural permet aisément l'accès à la nature. La maison individuelle est donc prisée. Pour développer démographiquement leur commune, les élus vont régulièrement s'appuyer sur la réalisation d'un lotissement qui propose des parcelles 
individuelles. Les habitations réalisées privilégient souvent la construction de plein pied. Or, la durabilité favorise l'économie des surfaces agricoles avec le principe de densification. Le lotissement durable de Delme a comme première conséquence de grignoter la SAU. Les parcelles constructibles à l'intérieur du village seraient à privilégier tout en y imposant des normes de constructions écologiques.

\section{2) Présentation et analyse du projet touristique de Marsal, respectueux de son passé}

92 La mare salée se situe au nord ouest du village, à l'extérieur des remparts.

Figure 15 : La mare salée est à proximité du village 12

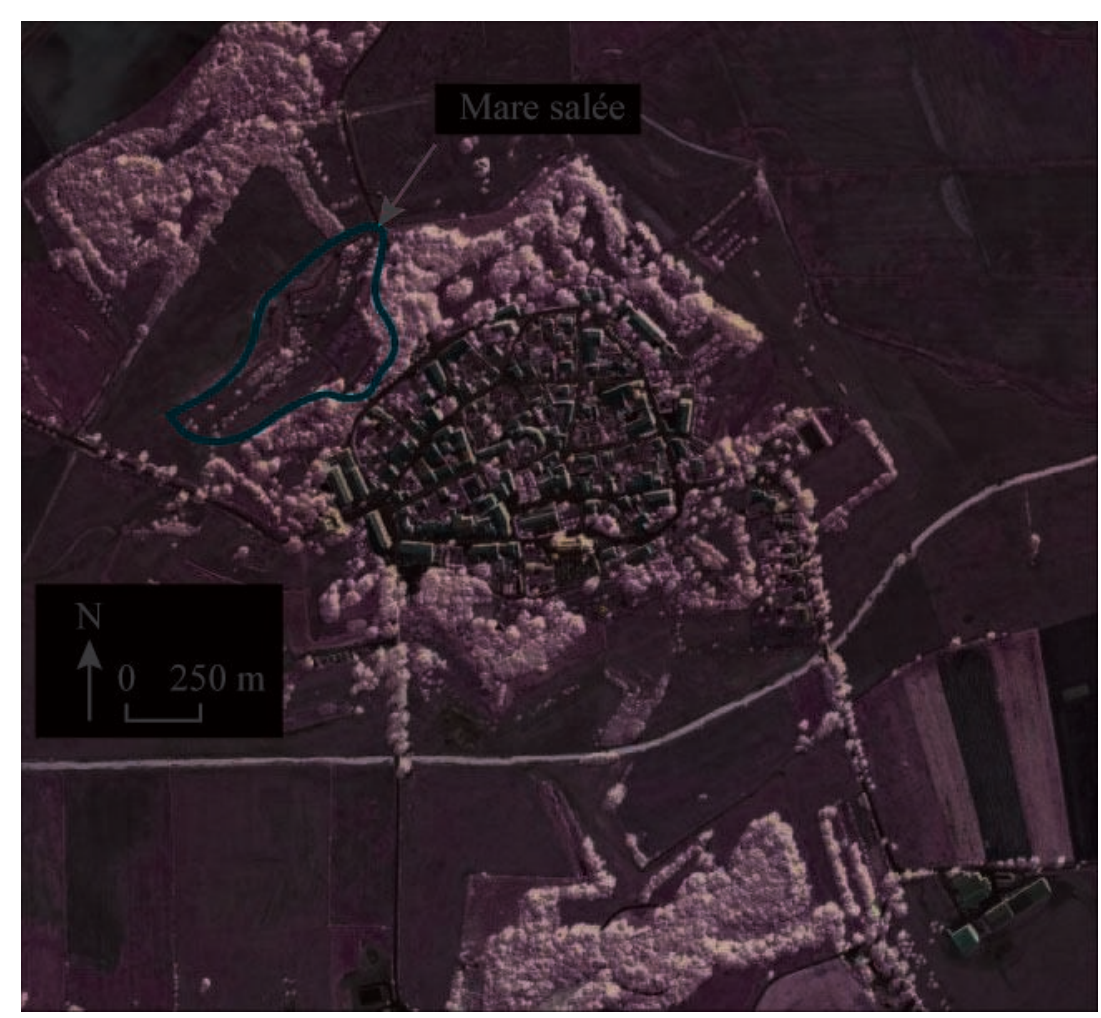

Dès la création du PNRL, une convention a été signée avec la commune pour la protéger car sa biodiversité est exceptionnelle (salicorne). Un premier aménagement fut réalisé. Une seule passerelle, visible sur la photographie, permettait d'accéder au cœur de la mare salée. Elle était en mauvaise état. 
Photographie 2 : Vue aérienne oblique de l'ancien aménagement de la mare salée ${ }^{13}$

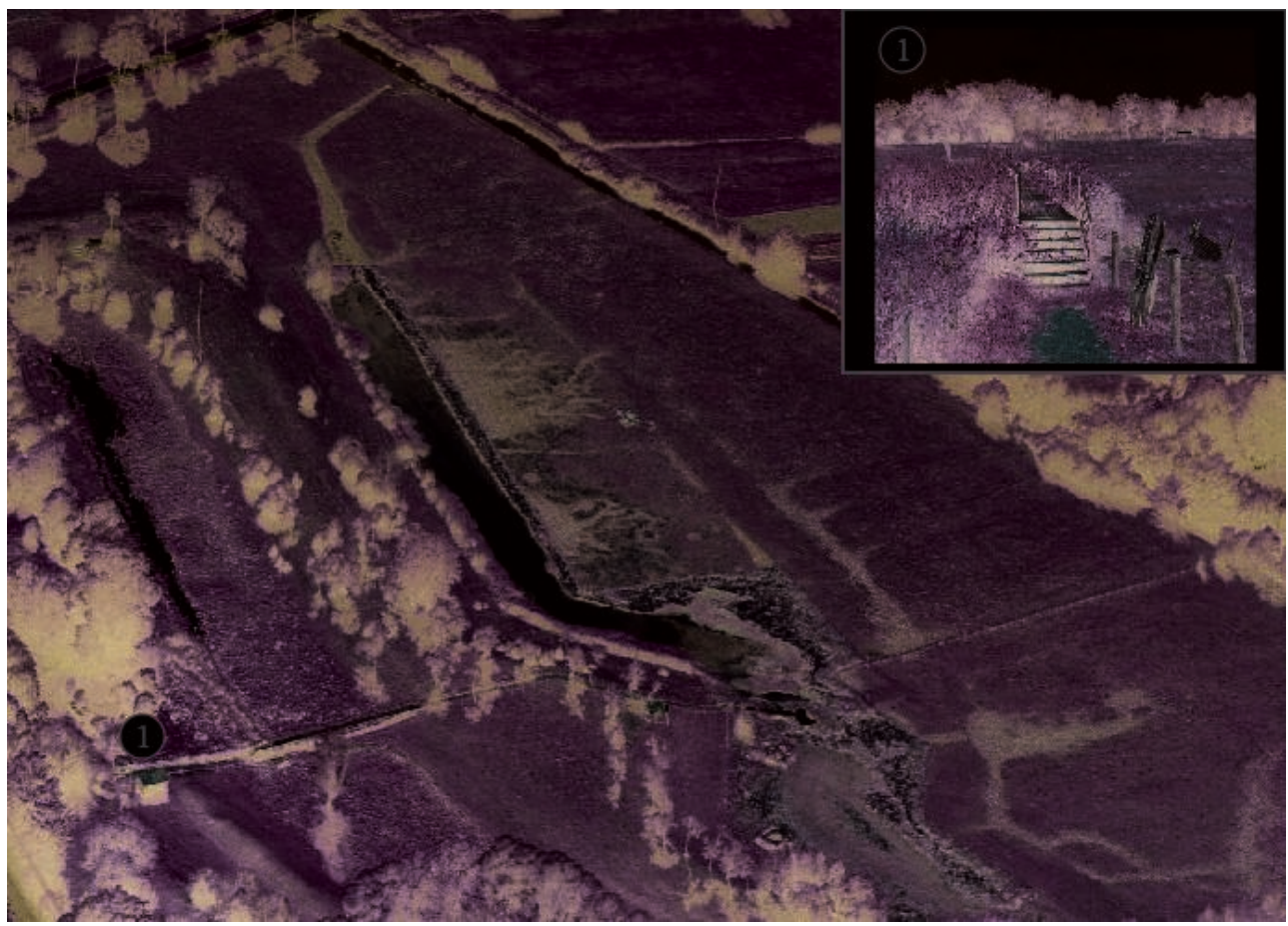

Source : Gille, 2013

94 Au premier plan, à gauche, les terrains des deux talus défensifs (à proximité du nombre 1), puis les mares salées dans leur environnement de prairies humides, adaptées à la présence du sel. Enfin, le lit d'inondation d'un bras de la Seille et la rectification géométrique du cours d'eau réalisée à l'époque allemande.

95 Le PNRL a souhaité réaménager ce site. Le parcours s'effectue à la fois sur deux passerelles et un sentier. Ils canalisent le trajet et n'abîment pas le milieu halophile. C'est par ces deux aménagements que les espèces faunistiques et floristiques sont protégées et valorisées. 
Photographie 3 : Le parcours aménagé de la mare salée ${ }^{14}$

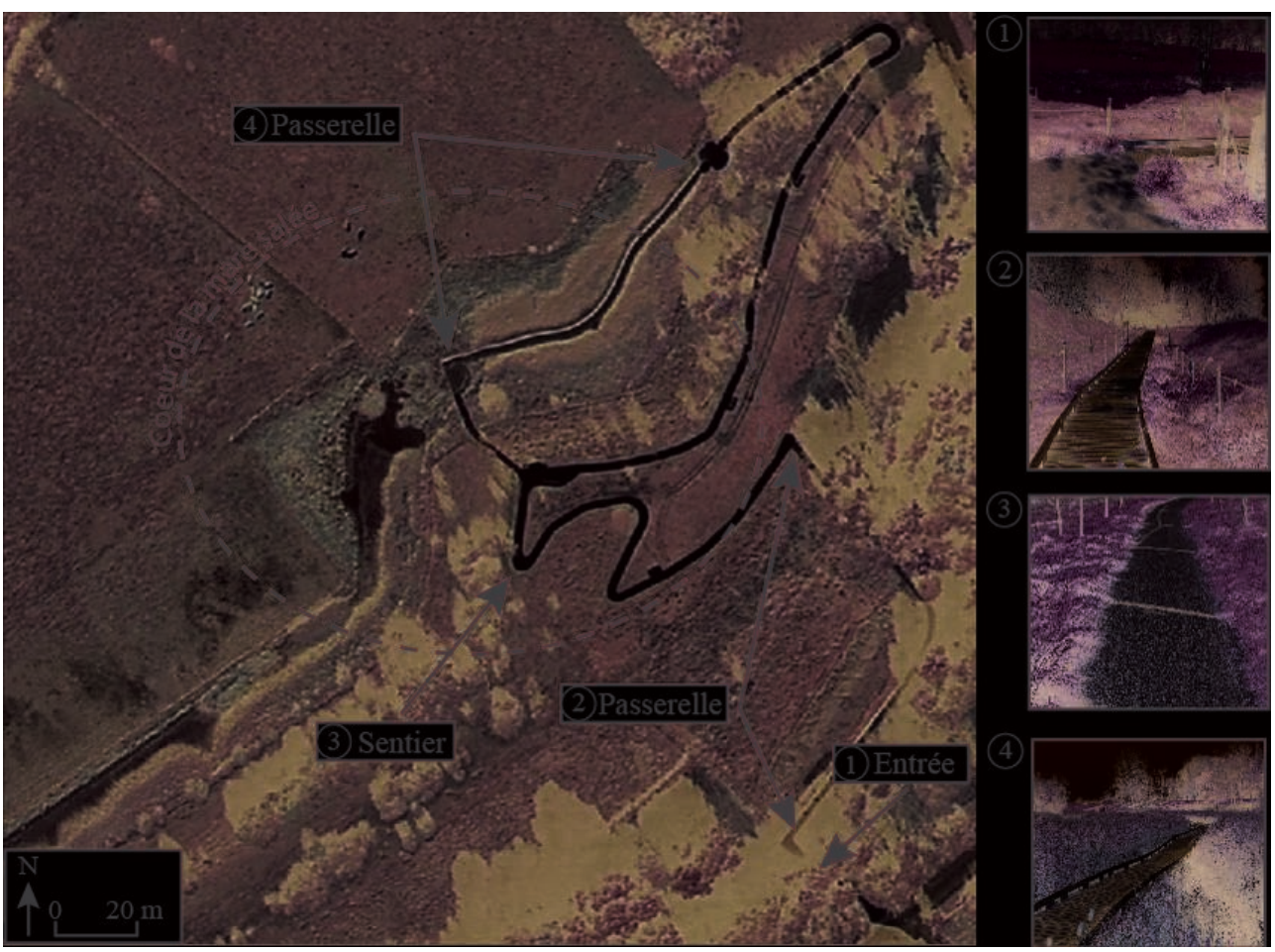

Source : Gille, 2013

96 Ce projet se doit d'appliquer les principes de gouvernance (consulter et associer les différents partenaires). Cette consultation et concertation existe par l'intermédiaire des différents courriers échangés entre les administrations. Concrètement, elle se traduit par différentes réunions organisées à Marsal pour suivre l'avancée du projet. La lecture des courriers d'invitation aux réunions révèle que la population et les associations protectrices de la biodiversité n'ont pas été conviées. Le projet d'aménagement reste une réussite. Ce projet revêt un intérêt touristique pour le développement de la commune. Il ne s'inscrit cependant pas dans une approche de Bottom-up qui s'impose progressivement. L'objectif unique du projet consiste à protéger la nature. Il n'applique en aucun cas l'ensemble des composantes du développement durable. Par exemple, il n'existe aucune communication sur ce projet dans le but de développer le tourisme et donc développer économiquement la commune. La gouvernance territoriale est de plus en plus présente dans les projets d'aménagement.

\section{La gouvernance territoriale}

La pratique de la gouvernance territoriale reste limitée puisque la participation de la société à l'aménagement du territoire est peu développée. Lors de l'élaboration d'un document d'urbanisme, la participation de la société civile reste limitée par deux actions, une législative et l'autre participative. Le code de l'urbanisme impose au conseil municipal, qui prescrit une carte communale, une concertation par le biais de l'enquête publique. Elle assure l'information et la participation du public. Les observations notées sont prises en considération. Les documents d'urbanisme qui ont 
des effets sur l'environnement ou qui affectent un site Natura 2000 doivent lancer une enquête publique. Le code de l'environnement énonce la procédure et le déroulement.

Dans le cadre de la carte communale de Marsal, l'enquête publique reste le principal moyen de concertation et de participation de la population. L'enquête publique est obligatoire car le projet impacte la zone Natura 2000.

L'enquête publique de Delme fut également obligatoire. Le projet inclut la réalisation de futures zones d'urbanisation (zone $\mathrm{AU}$ ) dont l'ouverture à la construction est prévue à moyen et long terme. Les habitants n'ont pas manifesté un véritable intérêt. Seules les personnes publiques associées ont souhaité quelques modifications mineures. Sur les deux enquêtes publiques observées, l'approche bottom-up (approche ascendante d'un projet où la réflexion démarre des perceptions de l'échelon le plus bas de l'échelle, c'est-à-dire de la population. Cette démarche favorise un pilotage participatif) qui consiste à partir n'existe absolument pas.

Dans le cadre de l'élaboration d'un PLU, les élus précisent les modalités de concertation avec la population pendant la durée du projet. Son bilan a lieu avant l'arrêt du projet. Le choix de Delme s'est orienté sur l'organisation, en fin de projet, d'une réunion publique pour présenter les objectifs du document d'urbanisme et ces effets. Cette réunion a rassemblé une petite partie des citoyens, essentiellement de vieux habitants du village. Elle a permis un échange entre les élus, le bureau d'études et les citoyens. Les citoyens associent parfois les réunions publiques à un moyen d'évoquer avec les élus les autres projets en cours sur la commune. Elles servent parfois à exprimer leur mécontentement.

1 Marsal a opté pour plusieurs solutions cadrant avec l'action participative. L'élaboration d'une carte communale n'impose pas la même mise en place de modalité de concertation. Les élus ont mis en place des actions participatives pour impliquer la société. Un cahier de doléances fut installé en mairie. Il était disponible aux heures d'ouverture. Les élus ont organisé une réunion publique avant l'organisation de l'enquête publique pour expliquer les raisons qui les ont poussées à se doter d'un document d'urbanisme. Ils ont expliqué le parti d'aménagement retenu. Les élus ont incité les habitants à se déplacer lors de l'enquête publique. L'échange a été l'occasion pour le maire de s'exprimer sur les montants de l'étude, sur les futurs projets et sur l'intérêt de protéger le patrimoine naturel et culturel de sa commune.

2 La mise en place des modalités de concertation reste à l'appréciation de la commune. Les choix diffèrent selon les territoires et les élus. La DDT assiste les élus ruraux pour élaborer le cahier des charges du PLU. Elle les conseille sur les possibilités de concertation mais manque d'imagination. La DDT fournit le même cahier des charges fournit aux communes qui souhaitent élaborer un document d'urbanisme. Il sert de base aux bureaux d'étude qui souhaitent répondent à l'appel d'offre. A leur lecture, la concertation repose sur deux éléments, la tenue de deux réunions publiques (en début de projet et avant l'enquête publique) au cours de l'élaboration du projet.

Elle n'explique pas l'intérêt majeur d'une telle procédure. Elle se contente de proposer les mêmes modalités de concertation sur les communes qu'elle assiste. Une volonté politique des élus explique des choix de concertation plus élaborés. Dans notre étude, nous nous situons dans un cas de gouvernance publique (Leroux, 2006). 


\section{Conclusion}

Le Sommet de Rio propose un nouveau mode de développement. La France l'impose progressivement à la conscience collective à travers sa législation en matière d'aménagement. Ce travail porte sur deux territoires géographiquement proches mais aux enjeux locaux différents.: Delme et Marsal. Elles aménagent leur territoire durablement à travers les agendas 21 locaux présents de facto avec les chartes qui régissent la vie du PNR et du Pays mais aussi avec les choix formulés par les élus.

propose des moyens juridiques variés pour élaborer de véritables documents de planification durable. La pratique usuelle demeure en deçà de la théorie. Les élus manquent de compétences techniques. Les partenaires publics n'assistent et ne conseillent pas assez les élus ruraux. Ces carences influencent la durabilité des projets.

Des élus sensibilisés peuvent être des leaders et porter ces projets. La faiblesse du système est, à la base, au niveau communal. Les communes rurales ne disposent pas de budgets conséquents. Des moyens financiers influencent les actions des collectivités vers des projets durables.

107 L'aménagement durable sur les deux communes reste sporadique et différent. L'application du développement durable est propre à chaque territoire (Mancébo, 2007, 2009). Il n'existe aucune coordination comme peut l'offrir un Agenda 21 local. La superposition des structures administratives, la multitude d'actions lancées par les entités supra-communales rendent difficilement lisible la politique d'aménagement durable. Les projets durables remettent parfois en cause les principes du sommet de 1992 à l'image du lotissement de Delme ou du projet de contournement routier qui réduisent la SAU. Le premier projet est pourtant un lotissement durable et le second s'inscrit dans la charte du pays considéré comme un Agenda 21 local.

108 L'élaboration d'un projet de territoire durable (sous la forme d'un Agenda 21 local) permettrait peut être de modifier les approches locales avec comme point de départ la coordination des politiques applicables à un territoire communal.

\section{BIBLIOGRAPHY}

Bailly A., 2009, Pour un développement rurbain durable, Revue d'économie régionale et urbaine, volume numéro 2, p. 231-238.

Barthelemy J., 1996, « Propos sur l'espace rural», Société géographique de Liège, hors série, p. 161-163.

Brundtland G., 1987, Notre avenir à tousCommission mondiale sur l'environnement et le développement, CMED, $349 \mathrm{p}$.

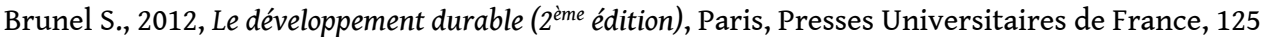

p.

Carlier B., 2012, Les Agendas 21, outils de développement durable, Voiron, Territorial Editions, 190 p. 
Cassin I., 2007, Le PLU : régime et champ d'application, Paris, Edition Le Moniteur, 242 p.

Chevallier J., 1993, « La gouvernance, un nouveau paradigme étatique ?», Revue française d'administration publique, volume numéro 1, p. 203-217.

Cormier L., De Lajarte A., Carcaud N., 2010, «La planification des trames vertes, du global au local : réalités et limites. ", Cybergeo, volume numéro 504, p. 1-24.

Dantonel-Cor N., 2011, Droit des collectivités territoriales, Clamecy, Lexifac, 303 p.

Decomp C., 2003, Itinéraires du patrimoine : Marsal, Nancy, Direction régionale des Affaires Culturelles de Lorraine, $36 \mathrm{p}$.

Decoster D-P., 2006, « La société civile au cœur de la gouvernance locale.», Les Cahiers de l'Urbanisme, volume numéro 62, p. 24-26.

Drobenko B., 2001, Droit de l'urbanisme : les conditions de l'occupation du sol et de l'espace, l'aménagement, le contrôle, le financement, le contentieux, Paris, Gualino, 315 p.

Ducroux AM. (dir),2002, Les nouveaux utopistes du développement durable, Paris, Édition Autrement, $342 \mathrm{p}$.

Emelianoff C., 2005, « Les agendas 21 locaux : quels apports sous quelles latitudes ?», Développement durable et territoire, volume numéro 4, p. 1-14.

Facène S., 2003, PLU de Delme, Document d'urbanisme, S-Péride, 156 p.

Facène S., 2004, PLU de Delme, Compte-rendu de réunion publique, S-Péride, 15 p.

Gilly J-P., Wallet F., 2005, Enchevêtrement des espaces de régulation et gouvernance territoriale. Les processus d'innovation institutionnelle dans la politique des Pays en France., Revue d'Économie Régionale et Urbaine, volume numéro 5, p. 699-722.

Gille F., 2008, Carte communale de Marsal, Compte-rendu de réunion publique, Terraxis, 3p.

Graindorge J., 2011, Grenelle 2, quels impacts pour les collectivités ?, Paris, Voiron, 88 p.

Jean B., Bisson L., 2008, «La gouvernance partenariale : un facteur déterminant du développement des communautés rurales ", Revue canadienne des sciences régionales, volume numéro 31, p. 539-560.

Laslaz L., Héritier S. (dir), 2008, Les parcs nationaux dans le monde : protection, gestion, et développement durable, Paris, Ellipses, 312 p.

Leloup F., Moyart L., Pecqueur B., 2005, « La gouvernance territoriale comme nouveau mode de coordination territoriale ?», Géographie, économie, société, volume numéro 7, p. 321-331.

Leroux E., 2012, « Le SCOT : un outil de Management public territorial au service du développement durable des territoires ?», Gestion et management public, volume numéro 1, p. 38-52.

Leroux I., 2006, «Gouvernance territoriale et jeux de négociation. Pour une grille d'analyse fondée sur le paradigme stratégique», Négociation, volume numéro 2, p. 83-98.

Lorach JM., De Quatrebarbes E., 2003, Guide du territoire durable : L'Agenda 21 territorial pour les collectivités locales et leurs partenaires, Paris, Village Mondial, $416 \mathrm{p}$.

Husson J-P., 2008, Envies de campagne : les territoires ruraux français, Paris, Ellipses, 207 p.

Husson J-P., Martin E., 2009, « Étude de géohistoire de la place forte de Marsal, analyse de l'hydrosystème de défense ", in Salzmann JP., Vauban et Marsal à l'époque de Louis XIV, le sel, la fiscalité et la guerre, Luxembourg, Institut Grand Ducal, $424 \mathrm{p}$. 
Kayser B., 1989, La renaissance rurale : sociologie des campagnes du monde occidental, Paris, Armand Colin, 316 p.

Landel P-A., Sénil N., 2009, Patrimoine et territoire, les nouvelles ressources du développement, Développement durable et territoires, p. 2-15.

Mancébo F., 2007, « Le développement durable en question (s).», Cybergeo, volume numéro 404, p. $1-12$.

Mancébo F., 2009, « Des développements durables. Quel référentiel pour les politiques de développement durable en Europe ?», Cybergeo, volume numéro 438, p. 2- 21.

Mancébo F., 2012, Développement durable, Paris, Armand Colin, 124 p.

Choay F., Merlin P. (dir), 2005, Dictionnaire de l'urbanisme et de l'aménagement, Paris, Presses universitaires de France, 963 p.

Ministère de l'Intérieur et de l'aménagement du territoire,2007, La décentralisation en mouvement, Paris : la Documentation française, $223 \mathrm{p}$.

Pays du Saulnois.,2002, Charte de territoire, document d'orientation, Pays du Saulnois,

$132 \mathrm{p}$.

Pays du Saulnois., 2008, Volet territorial, document d'orientation, Communauté de communes du Saulnois, $122 \mathrm{p}$.

Pays du Saulnois.,2008, CPER 2007-2013, document d'orientation, Communauté de communes du Saulnois, $40 \mathrm{p}$.

Perrier-Cornet P., 2002, «Intercommunalité, pays : les recompositions territoires », in A qui appartient l'espace rural ?, Editions de l'Aube, La tour d'Aigues, $141 \mathrm{p}$.

Plottu E., Plottu B., 2009, « Logiques territoriales et aménagement durable du territoire : Quelles règles de coordination et de décision ", Géographie, économie, société, volume numéro 11, p. 283-299.

PNRL., 2003, L'essentiel de la charte, document d'orientation, PNRL, 16 p.

Région Lorraine., 2011, Du Diagnostic stratégique au projet d'aménagement et de développement durable de la Lorraine, document d'orientation, Conseil Régional de Lorraine, $180 \mathrm{p}$.

Raymond R., 2009, « La société civile, ce nouvel acteur de l'aménagement des territoires », L'Information géographique, volume numéro 2, p. 10-28.

Schmit B., 2006, «L'aménagement et le développement durable au cœur des PLU », Revue Projet, volume numéro 291, p. 11-17.

Serre N., 2006, L'Agenda 21 pour un établissement éco-responsable, Besançon, Edition SCÉRÉN-CRDP Poitou-Charentes, $159 \mathrm{p}$.

Talbot D., 2006, « La gouvernance locale, une forme de développement local et durable ? Une illustration par les pays ", Développement durable et territoires, volume numéro 7, p. 2-15.

Tautelle F., 2010, « Vers la fin des Pays ? ", L'Information géographique, volume numéro 74, p. 17-28.

Theys J., 2002, « L'approche territoriale du développement durale, condition d'une prise en compte de sa dimension sociale », Développement durable et territoires, volume 1, p. 2-12.

Veyret Y. (dir),2007, Le développement durable, Paris, Edition Sedes, 432 p.

Vu B., 2008, Rénovation et Grenelle de l'environnement, Paris, Eyrolles, 111 p. 
Zaccaï E., 2001, « Définir le développement durable», Société géographique de Liège, volume numéro 41, p.17-25.

\section{Sites internet consultés}

CAUE, http://www.caue57.com/, 7 octobre 2014.

Communauté de communes du Saulnois, www.cc-saulnois.fr, 17 avril 2013-04-17

Conseil Général de Moselle, www.cg57.fr, 17 avril 2013.

DRAC Lorraine, http://www.culturecommunication.gouv.fr/Regions/Drac-Lorraine, 7 octobre 2014.

DREAL Lorraine, http://www.lorraine.developpement-durable.gouv.fr/carmen-a2693.html, 16 mars 2013

Géoportail, http://www.geoportail.gouv.fr, 20 mai 2013.

Légifrance, http://www.legifrance.gouv.fr, 2 avril 2013.

Mairie de Delme http://www.delme.fr, 3 avril 2013.

Ministère de l'Environnement et du développement durable, www.environnement.gouv.fr, 17 novembre 2012.

PNR Région Lorraine, http://www.pnr-lorraine.com, 17 mars 2013.

Ramsar, http://www.ramsar.org, 25 mai 2013.

Sillon lorrain, http://www.sillonlorrain.org, 2 juin 2013.

Vie publique, http://www.vie-publique.fr/decouverte-institutions/institutions/collectivitesterritoriales/principes-collectivites-territoriales/qu-est-ce-que-deconcentration.html, 7 octobre 2014

\section{NOTES}

1. Sillon lorrain, un réseau d'agglomération, http://www.sillonlorrain.org, 4 juin, 2013.

2. INSEE. (2012).- Evolution et structure de la population à Delme, RP1968 à 1990 dénombrements - RP1999 et RP2009 exploitations principales, Paris, p. 1.

3. INSEE. (2012)- Ibid.

4. Comité d'Histoire régional de Lorraine, http://www.chr-lorraine.fr/naudin/pop zoneA1_01.php?frm_ref_commune=1357\&frm_ref_zone=174, 17 mai 2013.

5. Fédération des parcs naturels régionaux de France, http://www.parcs-naturelsregionaux.tm.fr/fr/approfondir/les-parcs-et-la-loi.asp, 8/10/2015.

6. Lutte contre le changement climatique, préservation de la biodiversité et des milieux, cohésion sociale et solidarité entre les territoires et les générations, l'épanouissement de tous les êtres humains, créer une dynamique de développement suivant des modes de production et de consommation responsables.

7. Source : Cerpa n ${ }^{\circ}$ 95-0118, n 95-119, n95-120 et n' 95- 221.

8. En l'absence de règlement spécifique à l'intérieur de la carte communale, le Règlement National d'Urbanisme s'applique. On le trouve, dans le code de l'urbanisme, aux articles R 111-1 à R 111-24.

9. Géoportail, http://www.geoportail.gouv.fr/accueil, 7 juin 2013.

10. Géoportail, http://www.geoportail.gouv.fr/accueil, 7 juin 2013. 
11. Géoportail, http://www.geoportail.gouv.fr/accueil, 6 février 2015.

12. Géoportail, www.geoportail.gouv.fr, 1 juin 2013.

13. PNRL, http://www.pnr-lorraine.com/fr/heure-du-conte_E848146316.html, 31 mai 2013.

14. Géoportail, www.geoportail.gouv.fr,51 juin 2013 ; photographies : (2013).

\section{ABSTRACTS}

The summit of Rio, in 1992, created the notion of sustainable development in space planning. France put this notion in her planning policy. This paper asks the role of sustainable development in French rural planning ? To explain, we use two different territories: Delme and Marsal in Moselle. The actors who play a role in planning give solutions to do a new rural planning. The planning projects apply more or less the sustainable development.

Le sommet de la Terre, de 1992, intronise le développement durable dans l'aménagement du territoire. La France a transposé cette notion dans ses politiques d'aménagement. Ce travail s'interroge sur la place du développement durable dans l'aménagement des espaces ruraux français, à savoir de quelles manières s'applique-t-il sur les communes rurales désormais toutes réunies en Communauté de Communes? Pour illustrer ces propos, les exemples des communes de Delme et Marsal en Moselle sont développés. Pour ces petites communes assez novatrices, les différentes échelles de gouvernance territoriale fournissent des cadrages. Le lotissement durable, les projets de mise en valeur et de protection de la nature, la prise en compte de la durabilité dans les documents d'urbanisme participent plus ou moins à valoriser le développement durable.

Die Riokonferenz hat damals die nachhaltige Entwicklung gegründet. Frankreich hat dieses Konzept in seiner Raumordnungspolitik übertragen. Diese Arbeit stellt die Frage der Stelle der nachhaltigen Entwicklung in dem französischen ländlichen Raum, bzw wie führt sich die nachhaltige Entwicklung in der ländlichen Raumordnungspolitik aus ? Dafür benutzen wir zwei Beispiele : Delme und Marsal (Mosel). Die verschiedenen Ebenen der territorialen Leitung (Governance) bilden für diese kleinen innovativen Gemeinden einen Rahmen. Nachhaltige Siedlungsunterteilungen, Aufwertungsprojekte und Umweltschutz, sowie die Berücksichtigung der Nachhaltigkeit in den Stadtplanungsdokumenten tragen mehr oder weniger dazu bei, der nachhaltigen Entwicklung einen Mehrwert zuzuteilen.

\section{INDEX}

Mots-clés: développement durable, ruralité, planification territoriale, développement local Keywords: sustainable development, rural, territorial planning, local development Schlüsselwörter: nachhaltige Entwicklung, Ländlichkeit, Raumplanung, lokale Entwicklung 


\section{AUTHOR}

\section{FABIEN GILLE}

Doctorant contractuelfabien.gille[chez]univ-lorraine.fr / fabien.gille[chez]gmail.com Laboratoire Loterr (EA 7304), 23 Boulevard Albert 1er, BP 13397, 54015 Nancy Cedex 